\title{
Timed protocol analysis of interconnected mobile loT devices
}

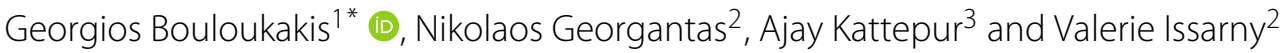

\section{*Correspondence:}

georgios.bouloukakis@telecomsudparis.eu

${ }^{1}$ Télécom SudParis, Institut Polytechnique de Paris, France Full list of author information is available at the end of the article

\begin{abstract}
With the emergence of the Internet of Things (IoT), application developers can rely on a variety of protocols and Application Programming Interfaces (APIs) to support data exchange between loT devices. However, this may result in highly heterogeneous IoT interactions in terms of both functional and non-functional semantics. To map between heterogeneous functional semantics, middleware connectors can be utilized to interconnect loT devices via bridging mechanisms. In this paper, we make use of the Data eXchange (DeX) connector model that enables interoperability among heterogeneous loT devices. DeX interactions, including synchronous, asynchronous and streaming, rely on generic post and get primitives to represent loT device behaviors with varying space/time coupling. Nevertheless, non-functional time semantics of loT interactions such as data availability/validity, intermittent connectivity and application processing time, can severely affect response times and success rates of DeX interactions. We introduce timing parameters for time semantics to enhance the DeX API. The new DeX API enables the mapping of both functional and time semantics of DeX interactions. By precisely studying these timing parameters using timed automata models, we verify conditions for successful interactions with DeX connectors. Furthermore, we statistically analyze through simulations the effect of varying timing parameters to ensure higher probabilities of successful interactions. Simulation experiments are compared with experiments run on the DeXMediators (DeXM) framework to evaluate the accuracy of the results. This work can provide application developers with precise design time information when setting these timing parameters in order to ensure accurate runtime behavior.
\end{abstract}

Keywords: Middleware, loT interactions, Interoperability, Timed automata, UPPAAL, Statistical analysis

\section{Introduction}

The Service Oriented Architecture (SOA) paradigm allows heterogeneous components to interact via standard interfaces by employing standard protocols. These are principally based on the client/server interaction paradigm, where typically a client sends a request to a server and gets the response within a timeout period. The successful completion of such an interaction depends on: $i$ ) the server's reachability; and $i i)$ the time needed to process the request, in comparison to the timeout period applied by the client.

(c) The Author(s). 2021 Open Access This article is licensed under a Creative Commons Attribution 4.0 International License, which permits use, sharing, adaptation, distribution and reproduction in any medium or format, as long as you give appropriate credit to the original author(s) and the source, provide a link to the Creative Commons licence, and indicate if changes were made. The images or other third party material in this article are included in the article's Creative Commons licence, unless indicated otherwise in a credit line to the material. If material is not included in the article's Creative Commons licence and your intended use is not permitted by statutory regulation or exceeds the permitted use, you will need to obtain permission directly from the copyright holder. To view a copy of this licence, visit http://creativecommons.org/licenses/by/4.0/. 
On the other hand, the advent of paradigms such as the Internet of Things (IoT) [1] involves not only conventional services but also sensor-actuator networks and data feeds. IoT applications consist of devices (i.e., Things) that may employ a variety of middlewarelayer protocols, such as MQTT, CoAP, ZeroMQ [2] and more, to interact with each other. These protocols may follow different interaction paradigms such as Client/Server (CS), Publish/Subscribe (PS), Data Streaming (DS), Tuple Space (TS), which determine the functional semantics of interactions. However, IoT applications are characterized by nonfunctional time semantics as well. In particular, exchanged data records may be valid or available for a limited lifetime (time-to-live) period. In addition, a considerable portion of IoT is mobile, which results to intermittently available data recipients. The latter, in conjunction with the data availability/validity, may affect the successful delivery of data in IoT applications.

The primary purpose of this paper is to model and analyze the aforestated time semantics in mobile IoT interactions. To deal with the Things' heterogeneity, we leverage the Data eXchange (DeX) connector model [3], which maps the end-to-end functional semantics of Things that employ heterogeneous middleware protocols. This is achieved via the DeX API that abstracts and unifies the four identified interaction paradigms (CS, PS, DS and TS) into four basic interaction types: (i) DeX one-way; (ii) DeX two-way sync; (iii) DeX two-way async; and (iv) DeX streaming (more details in Section 3). The DeX connector makes part of the DeXM framework, which enables interoperability among Things through the automated synthesis of mediators. In this paper, we extend the DeX API and connector model by introducing the following timing parameters: (i) lifetime and timeout to qualify the availability/validity in time of data or requests; (ii) serve_time to qualify the time it takes a server to process requests; and (iii) time_on/time_off to qualify the intermittent availability of data recipients. In our previous work [4], we modeled and analyzed the timing behavior of one-way interactions in service choreographies. In this paper, we generalize our modeling and analysis to one-way, two-way synchronous $\&$ asynchronous, and streaming interactions in IoT applications. This applies to systems that rely on not only any protocol following the CS, PS, DS and TS paradigms, but also any interconnection between them.

For our modeling and analysis, we use Timed Automata [5]. By relying on this formalism, we identify conditions for successful interactions and verify reachability and safety properties by employing the UPPAAL [6] model-checker. We further perform statistical analysis through the simulation of DeX interactions over multiple runs, and study the trade-off between interaction success rate and response time with varying timing parameters. To evaluate the accuracy of our simulation results, we compare them with experiments run with real protocol implementations on the DeX Mediators (DeXM) framework.

The approach introduced in this paper can be used by developers to compare between interaction types and paradigms, select among them, tune the timing parameters of the overlying application, and also do the previous when interconnection between heterogeneous protocols is involved. The main contributions of this paper are summarized as follows:

- We define timed $D e X$ interactions that represent basic end-to-end IoT interaction types enhanced with explicit time semantics. 
- We introduce formal timed models for DeX one-way, two-way synchronous \& asynchronous, and streaming interactions.

- We verify formal conditions for successful DeX interactions.

- We study the interaction success rate vs. response time trade-off with varying timing parameters through the simulation of DeX interactions.

- We validate our findings by running experiments with real protocol implementations.

The rest of the paper is organized as follows. We provide a motivating use case scenario and a high-level overview of our approach in Section 2. Then, in Section 3, we present the various semantics of DeX interactions, and introduce our enhancement of the DeX API. We also briefly describe the DeXM framework that interconnects IoT devices via mediators. The model for timing analysis of DeX interactions is introduced in Section 4. This is formalized with timed automata and related verification of properties in Section 5. The results of our analysis through simulation experiments are presented in Section 6, which includes comparison with experiments with the DeXM framework. This is followed by conclusions in Section 8.

\section{Overview}

Motivating Use Case. The detection and management of traffic congestion in a city is a critical issue in order to avoid significant delays while driving a vehicle [7]. For this purpose, several intelligent systems have been developed. We can classify them into three categories leveraging: (i) fixed-sensors (vehicle detectors, traffic cameras, doppler radars, etc) that have been installed on existing infrastructure [8,9]; (ii) vehicle (on-board) devices with GPS-based systems [10]; and, (iii) smartphones with embedded sensors (accelerometer, gyroscope) [11]. As depicted in Fig. 1, the combination of such intelligent systems can provide us an overall Transport Information Management (TIM) system in order to accurately estimate traffic conditions.

However, each of the above sensors/applications may rely on different protocol primitives, timing requirements and constraints when exchanging data as shown in Fig. 1. In particular, city-deployed, vehicle-device and smartphone sensed data are sent (periodically or not) to a broker system and then to the estimation service using the post primitive. To guarantee the freshness of provided information, notifications are maintained by the system for a (limited) lifetime period.

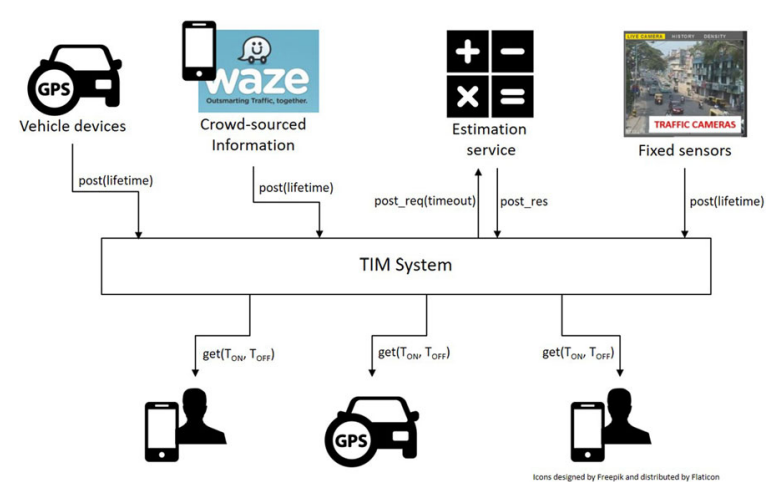

Fig. 1 Transport Information Management (TIM) system 
Mobile smartphones and vehicle devices access the system using the get primitive to receive up-to-date transport information. They stay connected for an average period $\left(\mathrm{T}_{\mathrm{ON}}\right)$ and then disconnect for resource saving purposes $\left(\mathrm{T}_{\mathrm{OFF}}\right)$. Disconnections occur due to resource saving purposes (e.g., battery), based on the budget of the mobile user's data plan or based on the networking issues of mobile data recipients. Finally, the estimation service processes the collected data and provides back the estimated traffic to smartphones/vehicle devices using the post_res primitive after their posted request (post_req) that has to be completed within a timeout period.

Given the above scenario, this work aims to address the following research questions: (i) despite the possibly high disconnection periods of mobile users, can a system designer ensure the timely delivery of traffic data to all recipients? (ii) can a system designer configure the connected periods of mobile users in order to guarantee successful and timely delivery of messages? (iii) can the validity of data be leveraged and configured to ensure specific delivery success rates of data?

High-Level Approach. Based on the primitives and timing constraints of the TIM system, an application designer should be able to analyze and configure certain system aspects (user connectivity, message lifetime period, etc) in order to guarantee the appropriate system response time and delivery success rate.

In this paper, we provide a time analysis approach as shows in Fig. 2 that enables:

1 Analysing the application's functional and time semantics, i.e., primitives and timing parameters, based on the employed middleware protocol(s) and the timing behavior of the IoT application.

2 Ensuring successful interactions in the IoT application by relying on the formal conditions identified using Timed Automata.

3 Performing statistical analysis using the DeX timing parameters for enabling system tuning.

Accordingly, system designers are able to redefine timing parameters of the TIM system and derive the corresponding formal conditions for successful interactions. Finally, by relying on our statistical analysis methodology, we demonstrate in Section 6 how system designers are able to tune the TIM system in order to ensure specific time requirements.

\section{Interconnecting heterogeneous things}

To enable the development of applications in IoT spaces, middleware IoT protocols, such as MQTT, CoAP, XMPP, etc, can be leveraged. These protocols provide a number of features such as supporting different Quality of Service (QoS) guarantees and they can be

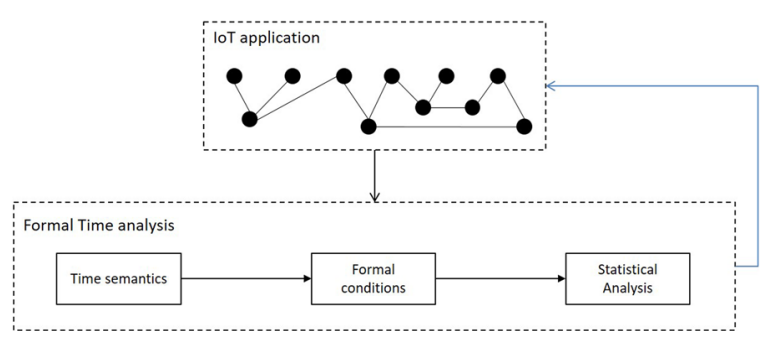

Fig. 2 Time Analysis Approach. Ensuring successful interactions into an loT application 
classified to an interaction paradigm (i.e., CS, PS, DS and TS). To express the different dimensions of coupling among communicating Things, we define the semantics of each interaction paradigm. We then leverage the DeX API [3], which is a set of primitives expressed as functions supported by the middleware. This API abstracts semantics of the basic interaction paradigms and therefore the semantics of the majority of existing IoT protocols. In this paper, we present a time-enhanced API that incorporates timing parameters of interactions such as timeout of requests, time validity of both messages (app-layer data) and control messages (e.g., subscriptions) and the Things intermittent connectivity. Finally, we explain how the DeX API is leveraged to model time-enhanced heterogeneous interactions in the IoT by relying on mediator software artifacts.

\section{1 loT semantics for data exchange}

By relying on [12-14], semantics of interest include space coupling, time coupling and synchronization coupling. Space coupling determines how Things identify each other and, consequently, how interaction elements (such as messages) are routed from one Thing to the other. Time coupling essentially determines if Things need to be present and available at the same time for an interaction or if, alternatively, the interaction can take place in phases occurring at different times. Finally, synchronization coupling determines whether the initiator of an end-to-end interaction blocks or not until the interaction is complete; in the former case, the interaction is executed in a synchronous way between the interacting Things. To express synchronization semantics, but also other semantics of end-to-end interactions, we define four interaction types and six role types for the interacting Things:

1 one-way interaction: a Thing can take either the sender or the receiver role. The sender sends a piece of data without waiting for a response; the receiver will asynchronously get notified for the arrival of the element by setting a listening \& callback mechanism.

2 two-way synchronous (sync) interaction: a Thing can take the client or server role. A synchronous interaction is blocking for the client and requires a prompt response from the server. Clients invoke a request on the server and then suspend their processing while they wait for a response for a specific timeout period.

3 two-way asynchronous (async) interaction: each Thing can take either the client or the server role. Clients initiate a request to a server and then continue their processing (non-blocking). The server handles the client's request using a callback and returns the response at some later point, at which time the client receives the response (also with a callback) and proceeds with its processing.

4 streaming interaction: a Thing can take either the consumer or the producer role. The consumer requests to establish a dedicated session with the producer. Once established, the producer sends multiple pieces of data that will asynchronously be received by the consumer. Depending on the middleware protocol, both Things or just the consumer can suspend, resume and terminate the session using the corresponding interaction elements.

As depicted in Fig. 3, middleware IoT protocols implement one or more of the above interaction types. For instance, in CS protocols, a client communicates directly with a server either by direct messaging (one-way) or by a remote procedure call (RPC, twoway) through an operation. In PS protocols, publishers publish events characterized by 


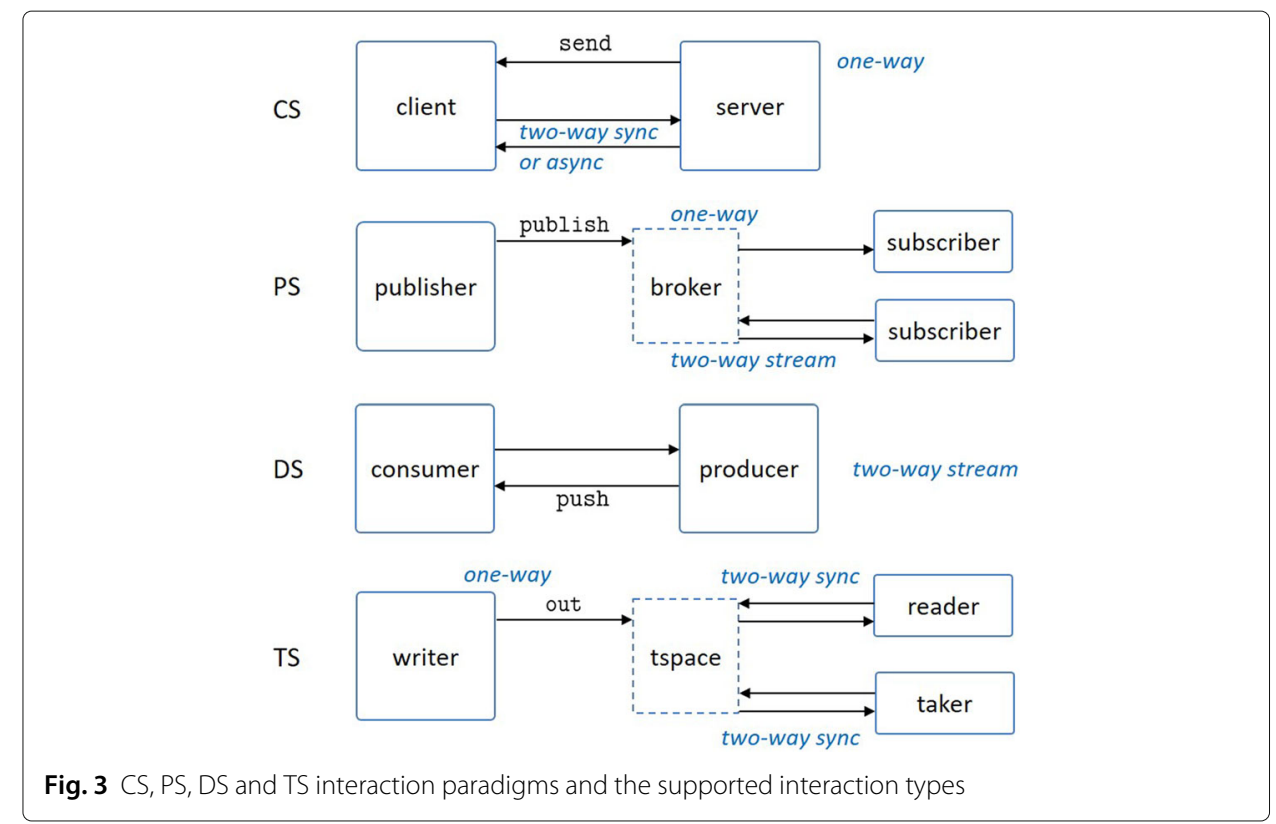

a specific filter (e.g., topic) to the broker. Subscribers may choose to check for pending events synchronously themselves (two-way synchronous) or set up a callback function that will be triggered asynchronously by the broker when an event arrives (streaming). In DS protocols, a consumer (typically) establishes a dedicated session with an open stream request sent to a producer. Upon the session's establishment, a continuous flow of data is pushed from the producer to the consumer. Finally, in TS protocols, multiple peers interact via an intermediate entity with a tuple space (tspace). Peers can write (out) data into the tspace and can also synchronously retrieve data from it, either by reading a copy or removing the data.

\subsection{Data eXchange (DeX) API}

The basic interaction types can be implemented using the corresponding library implementation of every IoT middleware protocol. In [3], we introduced the DeX connector that models the semantics of the majority of existing middleware protocols via the following high-level API primitives:

- (i) post employed by a Thing for sending data, also called messages, to one or more other Things.

- (ii) get employed by a Thing for receiving data.

In this paper, we enrich the DeX API [3] with the following timing parameters:

- lifetime: refers to emitted messages and characterizes both data availability/validity in time.

- timeout: allowed time period to complete a request-response synchronous interaction.

- time_on, time_off: instantaneous connected (ON) and disconnected (OFF) periods for receiving data.

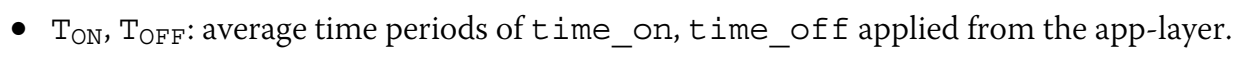


In Table 1, we present the enhanced primitives per interaction type. Below we provide a brief description:

DeX one-way. In a DeX one-way interaction a sender entity sends messages using the post primitive with a validity period lifetime. At the receiver side, the get primitive is used to enable the reception of messages through the get_return primitive. In addition, the receiving entity defines the average connection and disconnection time periods $\left(\mathrm{T}_{\mathrm{ON}}, \mathrm{T}_{\mathrm{OFF}}\right)$. Note that post and get operations are independent and have individual timestamps. We assume that application entities (undertaking the sender and receiver roles) enforce their semantics independently (no coordination).

DeX two-way sync. In a two-way synchronous interaction, a client entity posts requests using the post_req primitive and waits for timeout period to get the response via the get_res callback. At the server side, a thread identifier (thr_id) is assigned to the request procured through the get_req callback during the server's connected periods (i.e., $\left.\mathrm{T}_{\mathrm{ON}}\right)$. Subsequently, at the app-layer, the request is processed for serve_time and at the end of this period the server entity posts (post_res) the response associated with the same thr_id (unless the timeout period is reached). Finally, the message is delivered using get_res within the timeout period at the client side. In comparison to one-way interactions, client/server entities do not enforce their post and get semantics independently. In particular, after posting a request (post_req), the client is blocked to receive the response from the server using the get_res callback. On the other hand, requests and connection periods $\left(\mathrm{T}_{\mathrm{ON}} / \mathrm{T}_{\mathrm{OFF}}\right)$ are initiated independently from each other.

DeX two-way async. In a two-way asynchronous interaction, a client entity posts requests using the post_req primitive with a validity period lifetime. The response can be received at some point later through the get_res callback during the client's average connected periods $\mathrm{T}_{\mathrm{ON}}$. Similarly, at the server side, the request can be procured through the get_req callback during the server's average connected periods $\mathrm{T}_{\mathrm{ON}}$. Subsequently, the request is processed for serve_time at the app-layer and at the end of this period the server entity posts (post_res) the response with a lifetime validity period. Finally, if the message is not expired, it is then delivered using get_res at the client side. In comparison to two-way sync interactions, client/server entities enforce their post and get semantics independently. In particular, when a client post a request

Table 1 DeX primitives per interaction type

\begin{tabular}{|c|c|c|}
\hline Interaction & DeX primitives & \\
\hline \multirow[t]{2}{*}{ one way } & Sender & Receiver \\
\hline & post (lifetime) & $\begin{array}{l}\text { get }\left(\star \text { get_return, } \mathrm{T}_{\mathrm{ON}}, \mathrm{T}_{\mathrm{OFF}}\right) \\
\text { get_return }\end{array}$ \\
\hline \multirow[t]{2}{*}{ two way sync } & Client & Server \\
\hline & $\begin{array}{l}\text { post_req }\left(* g e t \_r e s, \text { timeout }\right) \\
\text { get_res }\end{array}$ & $\begin{array}{l}\text { get }\left(\star \text { get_req, } \mathrm{T}_{\mathrm{ON}}, \mathrm{T}_{\mathrm{OFF}}\right) \\
\text { get_req }(\text { thr_id) } \\
\text { post_res (thr_id) }\end{array}$ \\
\hline two way async & $\begin{array}{l}\text { post_req(lifetime) } \\
\left.\text { get (*get_res, } \mathrm{T}_{\mathrm{ON}}, \mathrm{T}_{\mathrm{OFF}}\right) \\
\text { get_res }\end{array}$ & $\begin{array}{l}\text { get }\left(\star \text { get_req, } \mathrm{T}_{\mathrm{ON}}, \mathrm{T}_{\mathrm{OFF}}\right) \\
\text { get_req } \\
\text { post_res (lifetime) }\end{array}$ \\
\hline \multirow[t]{2}{*}{ streaming } & Consumer & Producer \\
\hline & $\begin{array}{l}\text { post_open (lifetime, } \\
\text { flow_qualifier) get (*get_item, } \\
\left.\text { flow_qualifier, } \mathrm{T}_{\mathrm{ON}}, \mathrm{T}_{\mathrm{OFF}}\right) \\
\text {...get_item... }\end{array}$ & $\begin{array}{l}\text { get (*get_open, } \mathrm{T}_{\mathrm{ON}}, \\
\mathrm{T}_{\mathrm{OFF}} \text { ) } \\
\text { get_open (flow_qualifier) } \\
\text { \{...post_item(lifetime, } \\
\text { flow_qualifier)... }\end{array}$ \\
\hline
\end{tabular}


(post_req), it does not block its process to receive the response through get_res. Finally, requests and responses can be associated in the app-layer using identifiers that should be included in the parameters of post and get primitives.

DeX streaming. In a two-way streaming interaction, a consumer entity requests to establish an end-to-end connection with a producer using the post_open primitive. This connection is characterized using a flow_qualifier (i.e., a pair of $<$ producer, stream_id $>$ ) and is valid for lifetime period. Multiple data items can be received for that flow_qualifier at some point later through the get_item callback during the consumer's average connected periods $\mathrm{T}_{\mathrm{ON}}$. At the producer side, the stream can be established through the get_open callback during the producer's average connected periods $\mathrm{T}_{\mathrm{ON}}$. Subsequently, the producer entity posts (post_item) multiple data items with a lifetime and the flow_qualifier. In comparison to two-way async interactions, the producer entity enforce posts multiple items instead of a single response.

\subsection{DeX mediators}

We now present how timed DeX interactions can be implemented by relying on the DeX API. In particular, the DeX API can be leveraged from developers to implement the post and get primitives using existing IoT protocols such as CoAP, MQTT, XMPP, etc. Then, the DeX connector model defines the composition of different primitives for implementing DeX interactions to the so called DeX mediators (DeXM) [3]. As depicted in Fig. 4, the mediator converts traffic data coming from a vehicle device (in JSON format through the MQTT protocol) to be received from the estimation service (in XML format through the HTTP protocol). More details on DeXM can be found in [3].

While two heterogeneous Things rely on mediators to interact with each other, the resulting end-to-end interaction is one of the DeX interactions, i.e., one-way, two-way sync/async or streaming. Therefore, we can analyze the time semantics of Things using DeX to derive properties for ensuring successful interactions. We assume that the effect of the DeX mediators on processing/transmission delays of the end-to-end interactions is negligible. We intend to extend this model by relying on [15-17] to actually consider the timing effect of the DeX mediators.

In the next section we present the time model of DeX interactions.

\section{Time modeling of deX interactions}

In this section, we model DeX interactions with specific emphasis on their timing behavior [4]. We propose timing models that can represent end-to-end interactions of CS,

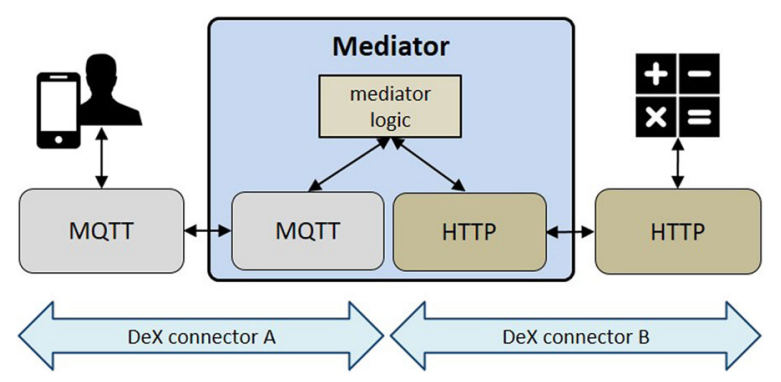

Fig. 4 Enabling timed DeX interactions via mediators 
Table 2 Analysis parameters' and shorthand notation

\begin{tabular}{ll}
\hline Parameter(s) & Definition/Description \\
\hline$t_{\text {post }}, t_{\text {get }}$ & at each timestamp $(t)$ one post or get occur. \\
$\delta_{\text {post }}, \delta_{\text {get }}$ & the time period between two successive post or \\
get operations. & message availability/validity in time. \\
lifetime & $\begin{array}{l}\text { connected (ON) and disconnected (OFF) } \\
\text { time_on, time_off }\end{array}$ \\
instantaneous periods for receiving messages. \\
time needed for a request to be processed at the \\
timeout & server side. \\
& required time period to complete a request-response \\
$t_{\text {post_req }}, t_{\text {get_req }}$ & synchronous interaction. \\
& $\begin{array}{l}\text { at each timestamp }(t) \text { one request is sent and } \\
\text { received, respectively. }\end{array}$ \\
$t_{\text {post_res }}, t_{\text {get_res }}$ & $\begin{array}{l}\text { at each timestamp }(t) \text { one response is sent and } \\
\text { received, respectively. }\end{array}$ \\
\hline
\end{tabular}

PS, DS and TS systems, but also any interconnection between them through DeXM, by relying on the DeX connector model. The parameters of our timing models are depicted in Table 2.

\subsection{One-way interactions}

We focus here on one-way interactions for CS, PS, DS and TS interaction paradigms represented by DeX. In particular, our analysis considers the "steady state" behavior of PS, DS and TS interactions. In PS, subscribers have been already subscribed to receive specific events when published and they do not unsubscribe during the study period. In DS, consumers have already established a session, and in TS readers/takers accessing the tuple space properly coordinate for preventing early removal of tuples by one of the peers before all interested peers have accessed these tuples.

As shown in Fig.5, in DeX one-way the post primitive is used to initiate the interaction at $t_{\text {post }}$; also called a post operation. A timer is started also at $t_{\text {post }}$, constraining the

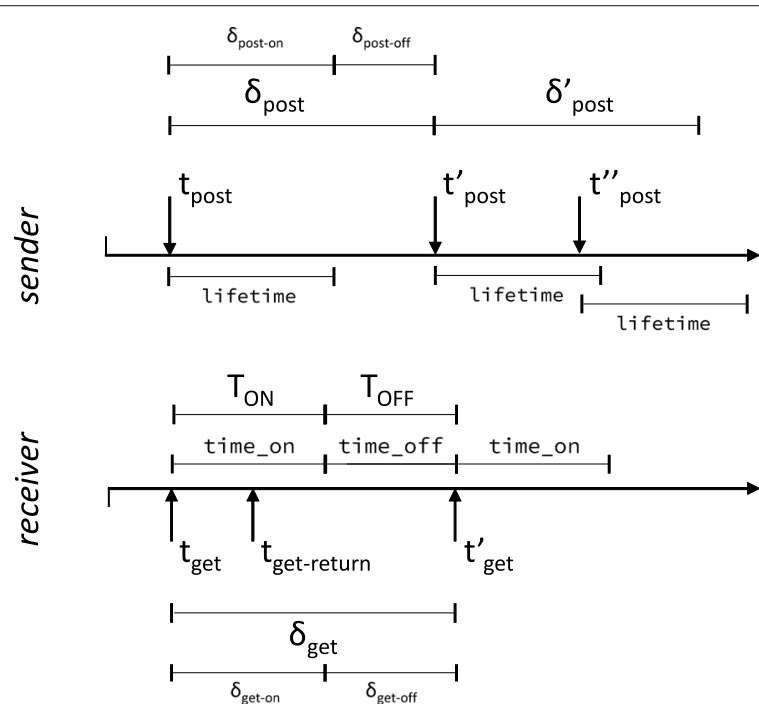

Fig. 5 Analysis of post and get $\delta$ increments for DeX one-way interactions 
message availability to the $l$ ifet ime period, also denoted by $\delta_{\text {post -on }}$. The period when the lifetime period elapses and the next post operation is yet to begin is denoted by $\delta_{\text {post-off }}$. Similarly at the receiver side, the get operation is initiated at $t_{\text {get }}$, together with a timer controlling the active period limited by the time_on (also denoted by $\delta_{\text {get-on }}$ ) interval. If get returns within the $t i m e \_$on period with valid data (not exceeding the lifetime), then the interaction is successful. We consider this instance also as the end of the post operation.

post operations are initiated repeatedly, with an interval rate $\delta_{\text {post }}$ (set as a random valued variable) between two successive post operations. Similarly, get operations are initiated repeatedly, with a random valued interval equal to $\delta_{\text {get }}$ between the start of two successive time_on periods; the interval between time_on and the next $t_{\text {get }}$ qualifies the disconnection period of receivers ( $t i m e \_$off or $\delta_{\text {get-off }}$ ).

While lifetime and time_on are in general set by application/middleware designers, inter-arrival delays $\delta_{\text {post }}$ and $\delta_{\text {get }}$ are stochastic random variables dependent on multiple factors such as concurrent number of peers, network availability, user (dis)connections and so on.

Note that this model allows concurrent post messages; buffers of active receiving entities (including the broker and tuple space) are assumed to be infinite, hence there is no message loss due to limited buffering capacity. The message processing, transmission and queueing (due to processing and transmission of preceding messages) times inside the interaction are assumed to be negligible compared to durations of $\delta_{\text {post }}$ and time_on periods.

In particular regarding queueing, we assume that we have no heavy load effects. This means that: all posts arriving during an active period are immediately served; all posts arriving during an inactive period are immediately served at the next time_on period, unless they have expired before. This corresponds to a G/G/ $\infty / \infty$ queueing model, where there are an infinite number of on-demand servers, hence there is no queueing. We assume that the general distribution characterizing service times incorporates the disconnections of receivers. We extend this model with actual queueing in [15-17].

Accordingly, successful one-way interactions depend on either of the disjunctive conditions:

$$
\begin{aligned}
& t_{\text {get }}<t_{\text {post }}<t_{\text {get }}+\text { time_on } \\
& t_{\text {post }}<t_{\text {get }}<t_{\text {post }}+\text { lifetime }
\end{aligned}
$$

meaning that a successful interaction occurs as long as a post and a get operation overlap in time. Otherwise, there is no overlapping in time between the two operations: only one of them takes place, and goes up to its maximum duration, i.e., lifetime for post and time_on for get.

Precisely:

1 If get occurs first, and then post occurs before time_on: the interaction is successful. Else, time_on is reached, and the get operation yields no interaction.

2 If post occur first, and then get occurs before lifetime: the interaction is successful. Else, lifetime is reached, and the interaction is a failure. 


\subsection{Two-way synchronous interactions}

In CS/TS two-way sync interactions, the client sends a request to a server and receives the response from the same server within a timeout period. The client's processing is blocked until the interaction is complete. As depicted in Fig.6, the post_req operation is initiated at $t_{\text {post_req. }}$. A timer is started also at $t_{\text {post_req }}$, constraining the requestresponse availability to the timeout period, also denoted by $\delta_{\text {post-req-on }}$. The period when the timeout period elapses and the next post_req operation is yet to begin is denoted by $\delta_{\text {post-req-off }}$. Similarly at the server side, the interval that allows to receive requests is initiated at $t_{\text {get }}$, together with a timer controlling the active period limited by the time_on (also denoted by $\delta_{\text {get-req-on }}$ ) interval. If get_req returns within the time_on period with a valid request (not exceeding the timeout), then after a serve_time interval (and only if it is still valid) the post_res returns the response to the client and the interaction is successful.

post_req operations are initiated repeatedly, with an interval rate $\delta_{\text {post }}$ (set as a random valued variable) between two successive post - req operations. Similarly, get operations are initiated repeatedly, with a random valued interval equal to $\delta_{\text {get }}$ between the start of two successive time_on periods; the interval between time_on and the next $t_{\text {get }}$ qualifies the disconnection period of receivers ( $t i m e$ off or $\delta_{\text {get-req-off }}$ ). timeout and time_on are in general set by application/middleware designers and inter-arrival delays $\delta_{\text {post }}$ and $\delta_{\text {get }}$ are stochastic random variables (dependent on network availability, user (dis)connections, etc).

Similar to the one-way timing model, concurrent post requests are allowed; (there is no message loss due to limited buffering capacity). However, once a post_req is active, the client blocks its operation and waits for the response during timeout. The requestresponse transmission and queueing times are assumed to be negligible compared to durations of $\delta_{\text {post }}$ and time_on periods. On the other hand, the processing of requests on the server side is defined using the serve_time interval.

Successful interactions depend on the following condition:

$$
t_{\text {post_req }}<t_{\text {get }}+\text { serve_time }<t_{\text {post_req }}+\text { timeout }
$$

meaning that a successful interaction occurs as long as: (i) a post_req and a get operation overlap in time; and (ii) when there is an overlap between the post_req and

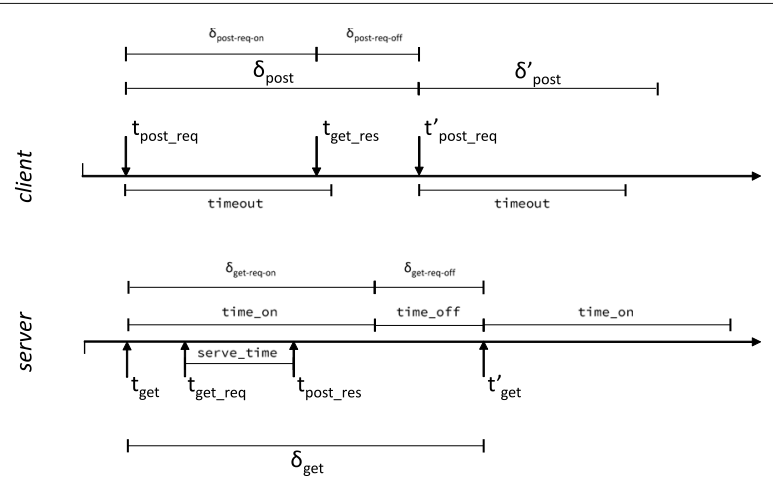

Fig. 6 Analysis of post and get $\delta$ increments for two-way synchronous interactions 
the get operations, the request must be served before the timeout period is reached. Otherwise, there is no overlapping in time between the two operations: only one of them takes place, and goes up to its maximum duration, i.e., timeout for post_req and time_on for get.

Failed interactions occur in the following cases:

1 When post_req occurs, and then get occurs before timeout: the get_req is enforced. Else, timeout is reached, and the interaction results in a failure.

2 After enforcing the get_req operation, if the get_res occurs before timeout: the interaction is successful. Else, the timeout is reached due to the processing at the server side, and the interaction results in a failure.

\subsection{Two-way asynchronous interactions}

The timing behavior of two-way async and streaming interactions can be represented by relying on the time model for one-way interactions presented in subsection 4.1. Asynchronous interactions are non-blocking operations where a client sends a request to a server and then resumes its processing without waiting for a response. Based on Table 1, two-way async interactions are bidirectional where each direction is modeled using post and get primitives as follows: (i) a client posts a request assigned with lifetime using the post_req primitive and the server uses the get primitive to receive the request through the get_req callback; (ii) the server posts the response assigned with lifetime using the post_res primitive and the client uses the get primitive to receive the response through the get_res callback. Note that two-way sync interactions are bidirectional as well, however, client/server entities do not enforce their post and get primitives independently.

Accordingly, successful two-way async interactions depend on the following disjunctive conditions:

$$
\begin{aligned}
& t_{\text {get }}<t_{\text {post_req }}<t_{\text {get }}+\text { time_on } \\
& t_{\text {post_req }}<t_{\text {get }}<t_{\text {post_req }}+\text { lifetime } \\
& t_{\text {get }}<t_{\text {post_res }}<t_{\text {get }}+\text { time_on } \\
& t_{\text {post_res }}<t_{\text {get }}<t_{\text {post_res }}+\text { lifetime }
\end{aligned}
$$

where any request condition can be combined with one of the response conditions for successful interaction. At $t_{\text {post_req }}$ only requests are posted and get primitives with connectivity parameters are initiated at the server side (Eqs. 4, 5). Otherwise, at $t_{\text {post_res }}$ only responses are posted and get primitives with connectivity parameters are initiated at the client side (Eqs. 6, 7).

\subsection{Streaming interactions}

Similar conditions for successful DeX streaming interactions can be derived from the conditions for successful two-way asynchronous interactions. In particular, while in asynchronous interactions a single response (message) can be followed by one request, in 
streaming interactions there can be multiple unlimited responses. Based on Table 1, streaming interactions are bidirectional where each direction is modeled using the oneway post and get primitives as follows: (i) the consumer requests to open a stream using the post_open primitive assigned with the lifetime parameter. Then, the producer uses the get primitive to receive the open stream request through the get_open callback; ( $i$ ) the producer posts multiple responses assigned with lifetime using the post_item primitive and the consumer uses the get primitive to receive multiple responses through the get_item callback.

By relying on the timing models presented in this section, we can cover the various interaction types found in the IoT and represent the individual CS, PS, DS and TS paradigms, but also any heterogeneous interconnection between them, e.g., a PS subscriber interacting with a DS producer via DeX mediators.

In our future work, we aim to exploit our experience in the modeling of publish/subsbribe protocols using queueing theory [15-17] to relax the assumptions (queueing/transmission delays, message losses) of the current model. In particular, we intend to leverage features such as limited capacity, ON/OFF connectivity intervals, heterogeneous arrival rates, etc., and apply them to queueing models. The resulting queueueing networks can evaluate realistically the performance of heterogeneous IoT interactions.

\section{Timed automata-based analysis}

As we have analyzed the timing behaviour of DeX one-way and two-way interactions, the next step is to formally guarantee correctness of the implemented solutions. This is particularly needed in the case of IoT systems as there are varied interaction patterns with timing constraints that could be affected by incorrect parameter setting. To verify safety and reachability properties of these interactions, we make use of model checkers. Using the expressive nature of some of these models, we can include stochastic delays along with deterministic time bounds to formally model the interactions. This further allows to guarantee absence of deadlocks or livelocks within the system, which is difficult to estimate via simulations or data analysis.

In this section, we build timed automata models which represent the typical behavior of the DeX connector model for performing the timed DeX interactions described in the previous section. A timed automaton [5] is essentially a finite automaton extended with real-valued clock variables. These variables model the logical clocks in the system, which are initialized with zero when the system is started, and then increase synchronously at the same rate. Clock constraints are used to restrict the behavior of the automaton. A transition represented by an edge can be taken only when the clock values satisfy the guard labeled on the edge. Clocks may be reset to zero when a transition is taken. Clock constraints are also used as invariants at locations, which are represented by vertices: they must be satisfied at all times when the location is reached or maintained.

In order to study DeX interactions with timed automata, we make use of UPPAAL [6]. UPPAAL is an integrated tool environment for modeling, validation and verification of real-time systems modeled as networks of timed automata. In such networks, automata synchronize via binary synchronization channels. For instance, with a channel declared as chan $\mathrm{c}$, a transition of an automaton labeled with $\mathrm{c}$ ! (sending action) synchronizes with the transition of another automaton labeled with $c$ ? (receiving action). UPPAAL makes use of computation tree logic (CTL) [18] to specify and verify temporal logic properties. 
We employ the committed location qualifier (marked with a ' $\mathrm{C}$ ') for some of the locations. In UPPA AL, time is not allowed to pass when the system is in a committed location; additionally, outgoing transitions from a committed location have absolute priority over normal transitions. The urgent location qualifier (marked with a ' $U$ ') is also used: time is not allowed to pass when the system is in an urgent location (without the priority clause of committed locations, though).

By relying on the expressive power of timed automata, we are able not only to model the timing conditions of DeX interactions, but also to introduce basic stochastic semantics regarding the behavior of peers. Using the UPPAAL model checker, we provide and verify essential properties of our timed automata model, including formal conditions for successful DeX interactions. Note that the time to verify the properties in UppAAL is on the scale of one second maximum.

\subsection{Analysis of one-Way interactions}

We represent one-way DeX interactions with the connector roles DeX sender, DeX receiver, and with the corresponding DeX one-way glue. The two roles model the behavior expected from application components employing the connector, while the glue represents the internal logic of the connector coordinating the two roles. We detail in the following the modeling of these components.

Figure 7 shows the sender behavior. Typically, a sender entity repeatedly emits a post ! action (message) to the glue without receiving any feedback about the end (successful or not) of the post operation. We have enhanced (and at the same time constrained) the sender's behavior with a number of features. The committed locations post_event (post! sent to the glue) and post_end_event (post_end? received from the glue) have been introduced to detect the corresponding events. Upon these events, the automaton oscillates between the post_on and post_off locations, which correspond to the $\delta_{\text {post-on }}$ and $\delta_{\text {post-off }}$ intervals presented in Fig. 5 . delta_post is a clock that controls the $\delta_{\text {post }}$ interval between two successive post operations. delta_post is reset upon a new post operation and set to lifetime at the end of this operation (note that the post_init location and its outgoing transition serve to initialize delta_post at the beginning of the sender's execution - this unifies verification also for the very first post operation). The invariant condition delta_post<=max_delta_post (where

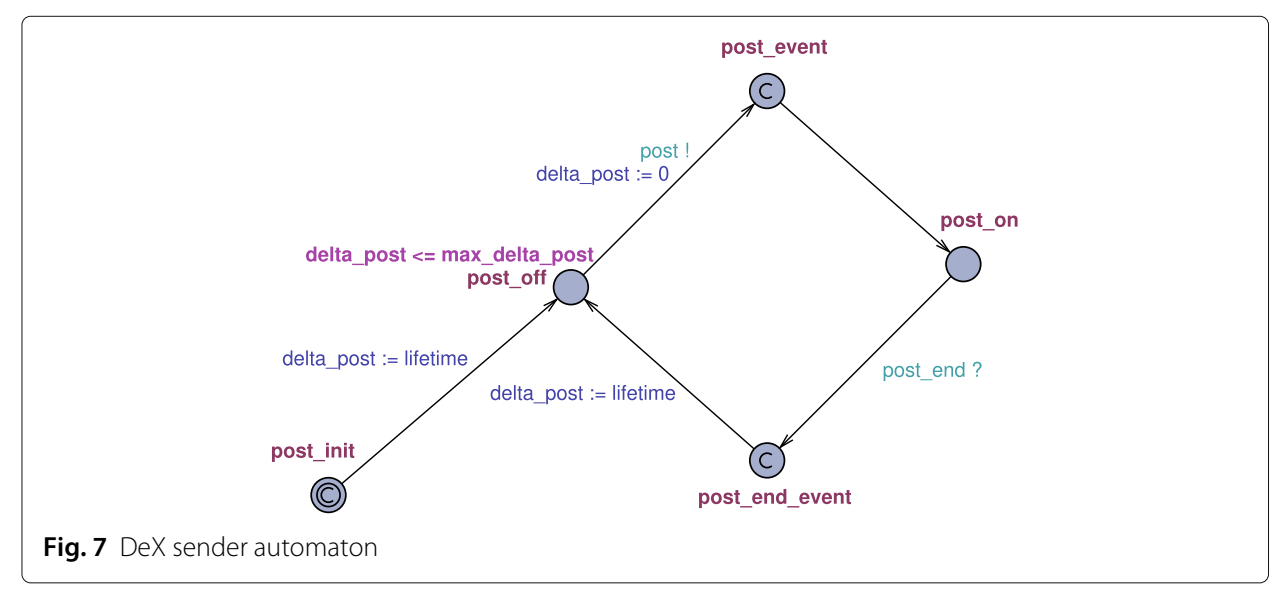


max_delta_post is a constant) at the post_off location ensures that a new post operation will be initiated before the identified boundary.

This setup results in at most one post operation active at a time. This post remains active ( $\delta_{\text {post-on }}$ interval) for lifetime interval (and then it expires) or less than lifetime interval (in case of successful interaction). In both cases, we set delta_post to lifetime at the end of the post operation (this enables verification, since we can not capture absolute times in UPPAAL). Hence, the immediately following $\delta_{\text {post - off }}$ interval will last a stochastic time uniformly distributed in the interval [lifetime,max_delta_post]. With regard to the one-way timing model of Section 4, we opted here for restraining concurrency of post operations for simplifying the architecture of the glue. The present model (sender, receiver and one-way glue) can be compared to one of the infinite on-demand servers of the G/G/ $\infty / \infty$ model of Section 4. Nevertheless, this model is sufficient for verifying Conditions (1) and (2) for successful DeX interactions. These conditions relate any post operation with an overlapping get operation; possible concurrency of post operations has no effect on this. Moreover, in the following sections we prove that these conditions are independent of the probability distributions characterizing the sender and receiver's stochastic behavior.

Figure 8 shows the receiver behavior. Typically, a receiver entity repeatedly emits a get! action to the glue, with at most one get operation active at a time. The duration of the get operation is controlled by the receiver with a local time_on; upon the time_on, a get_end! action is sent to the glue. Before reaching the time_on, multiple messages (posted by senders) may be delivered to the receiver by the glue, each with a get_return? action. We have enhanced the receiver's behavior with similar features as for the sender. Hence, we capture the events and time intervals presented in Fig. 5 with the get_event, get_end_event, get_on, get_off locations, as well as with the delta_get clock and the invariant conditions delta_get<=time_on (at get_on) and delta_get<=max_delta_get (at get_off). This setup results in a succession of $\delta_{\text {get-on }}$ and $\delta_{\text {get-off }}$ intervals, with the former lasting time_on time and the latter lasting a stochastic time uniformly distributed in the interval [time_on,max_delta_get]. We have additionally introduced the committed location no_trans, which, together with the Boolean variable get_ret, helps detecting whether the whole time_on period elapsed with no interaction performed or at least one message was received.

The glue one-way automaton is shown in Fig. 9. It determines the synchronization of the incoming post? and get? operations. A successful synchronization between such

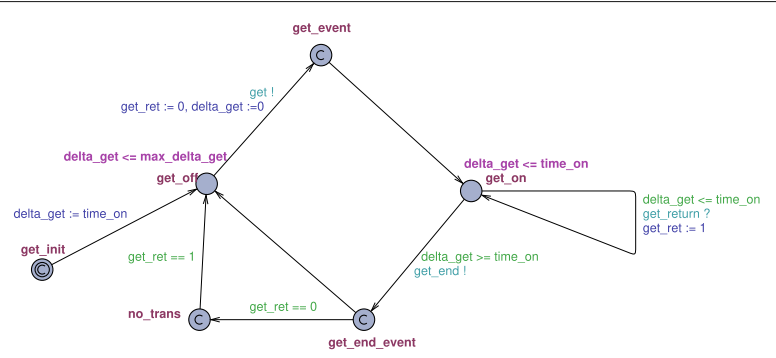

Fig. 8 DeX receiver automaton 


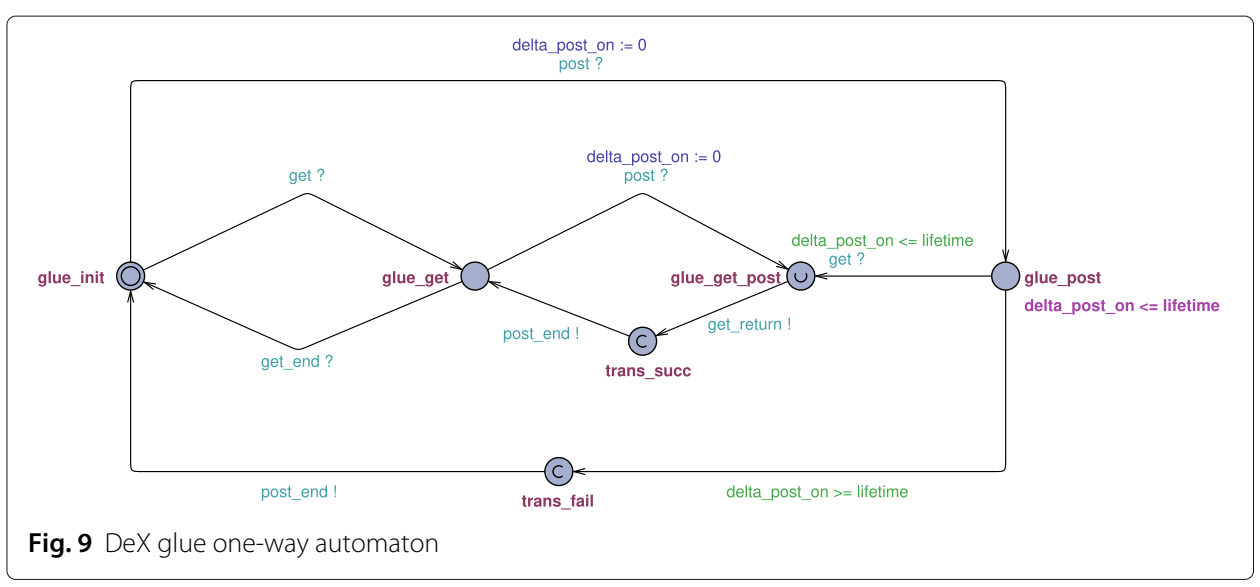

operations leads to a successful interaction, which is represented in the automaton by the trans_succ location. Note that the timing constraints specified in Section 4 regarding the lifetime of posted messages have been applied here with the additional clock delta_post_on employed to guard transitions dependent on the lifetime period. Two ways for reaching the trans_succ location are considered:

- If the get? operation occurs from the initial location (leading to location glue_get), a consequent post? operation results in a get_return! message and eventually the successful interaction location trans_succ (Eq. 1). At the same time, the sender is notified of the end of the post operation with post_end!. Note that we employ the urgent location qualifier for glue_get_post; thus, the glue completes instantly the successful interaction and is ready for a new one. At the glue_get location, if the get_end? action is received from the receiver automaton (suggesting delta_get $>=$ timeout), the glue is reset to the initial location glue_init.

- If the post? operation occurs initially (leading to location glue_post), a get? operation before the constraint delta_post_on $<=$ lifetime results again in a successful interaction (Eq. 2). Exceeding the lifet ime period without any get? results in location trans_fail, and the automaton returns to its initial location glue_init, notifying at the same time the sender with post_end!. This is done without any delay, thanks to the invariant delta_post_on $<=$ lifetime at the glue_post location.

\subsubsection{Verification of properties}

We verify reachability and safety properties of the combined automata DeX sender, DeX receiver and DeX glue one-way, by using the model checker of UPPAAL. A reachability property, specified in Uppaal as $\mathrm{E}<>\varphi$, expresses that, starting at the initial state, a path exists such that the condition $\varphi$ is eventually satisfied along that path. A safety property, specified in UPPAAL as A [ ] $\varphi$, expresses that the condition $\varphi$ invariantly holds in all reachable states.

Sender Automaton. We verify a set of reachability and safety properties that characterize the timings of the sender's stochastic behavior. 
A[] sender.post_event imply delta_post $==0$

A[] sender.post_on imply delta_post<=lifetime

A[] sender.post_off imply (delta_post>=lifetime and

$$
\text { delta_post<=max_delta_post ) }
$$

$\mathrm{E}<>$ sender.post_end_event and delta_post< lifetime

Equation 8 states that post events occur at time 0 captured by the delta_post clock. Equation 9 and 11 together state that [0,1 ifetime] is the maximum interval in which a post operation is active; nevertheless, the operation can end before lifetime is reached. Equation 10 states that [lifetime,max_delta_post] is the maximum interval in which there is no active post operation. This confirms the fact that we artificially "advance time" to lifetime at the end of the post operation.

Receiver Automaton. We verify similar properties that characterize the timings of the receiver's stochastic behavior.

$$
\begin{aligned}
& \text { A [] receiver.get_event imply delta_get }==0 \\
& \text { A[] receiver.get_on imply delta_get<=time_on } \\
& \text { A[] receiver.get_off imply (delta_get>=time_on and } \\
& \text { delta_get<=max_delta_get) } \\
& \text { A[] receiver.get_end_event imply delta_get==time_on }
\end{aligned}
$$

Hence, Eq. 12 states that get events occur at time 0 captured by the delta_get clock. Equation 13 and 15 together state that a get operation precisely and invariantly terminates at the end of the [0,time_on] interval. Equation 14 states that [time_on,max_delta_get] is the maximum interval in which there is no active get operation.

Glue one-way Automaton. We verify conditions for successful interactions using the glue automaton.

$$
\begin{aligned}
& \text { A[] glue.trans_succ imply (sender.post_on and receiver.get_on } \\
& \text { and (delta_post }==0 \text { or delta_get }=0 \text { )) }
\end{aligned}
$$

In addition to the reachability property ( $E<>$ glue.trans_succ), we verify the safety property in Eq. 16. According to this, a successful interaction event implies that while a post operation is active a get event occurs, or while a get operation is active a post event occurs.

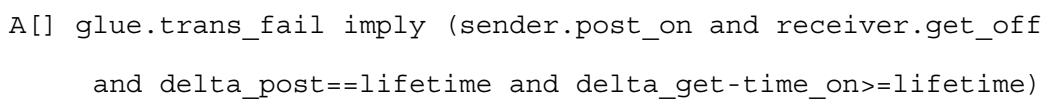

We verify both the reachability property ( $E<$ glue.trans_fail) and the safety property in Eq. 17. A failed interaction event means that lifetime is reached for an active post operation and no get operation is active. Additionally, the ongoing inactive get interval entirely includes the terminating active post interval. With regard to the stochastic post and get processes of our specific setting, we explicitly checked that if the 
condition max_delta_get-time_on>=lifetime does not hold for the given values of the included constants, then the reachability property $E<>$ glue.trans_fail is indeed not satisfied.

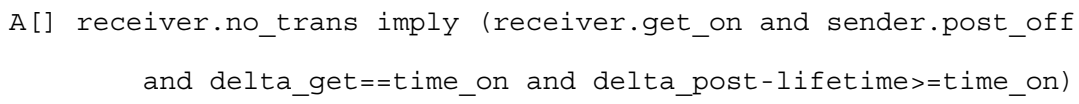

We verify both the reachability property ( $\mathrm{E}<>$ receiver.no_trans) and the safety property in Eq. 18. Symmetrically to Eq. 17, a no-interaction event implies that time_on is reached for an active get operation and no post operation is active. Additionally, the ongoing inactive post interval entirely includes the terminating active get interval. Similarly to Eq. 17, we check that if this safety property is not satisfied, then the state receiver.no_trans is indeed not reachable.

Observing Eqs. 16, 17, 18, we see (as intuitively expected) that successful, failed and no-interactions are determined by the durations and relative positions in time of the $\delta_{\text {post-on }}, \delta_{\text {post-off }}, \delta_{\text {get-on }}$ and $\delta_{\text {get-off }}$ intervals. These depend on the deterministic parameter constants lifetime, time_on and on the stochastic parameters $\delta_{\text {post }}$ and $\delta_{\text {get }}$. It is also worth nothing that Eqs. 16, 17, 18 are expressed in a general way, independently of the specific post and get stochastic processes. For example, Eq. 17 states that, to have a failed interaction, a lifetime period must be lower than the time_off period. But time_off is probabilistic. Therefore, to avoid failed interactions, a system designer can tune the system by changing (or trying to affect) these parameters accordingly, while they can employ any probability distribution for the disconnection parameter. Similarly, Eq. 18 provides the developer with hints of how to possibly avoid no-interactions. Hence, the analysis results of this section provide general formal conditions for successful DeX interactions and their reliance on observable and potentially tunable system and environment parameters. Using these results, we perform experiments to quantify the effect of varying these parameters for successful interactions in Section 6.

\subsection{Analysis of two-Way synchronous interactions}

We represent two-way synchronous DeX interactions with the connector roles DeX client, DeX server, and DeX two-way sync glue. We detail in the following the modeling of these components.

Figure 10 shows the client behavior. Typically, a client emits a post_req (request) to the glue and waits for timeout to receive the get_res (response). The committed location post_req_sent is introduced to detect the event of sending a request (post_req!) to the glue. Upon such an event, the automaton stays on the post_req_on location to either receive the response or until the timeout expires, which corresponds to the $\delta_{\text {post-req-on }}$ interval presented in Fig. 6 . Upon the timeout expiration or the get_res reception, the automaton stays in the post_req_off for $\delta_{\text {post-req-off time period. }}$

delta_post is a clock that controls the $\delta_{\text {post }}$ interval between two successive post_req operations. delta_post is reset upon a new post_req operation and set to timeout upon a get_res? (prior to the timeout expiration). On the other hand, when the timeout period is reached, the delta_post clock is already set to timeout on the post_req_off location. We initialize delta_post at the beginning of the client's execution (post_init location). The invariant condition 


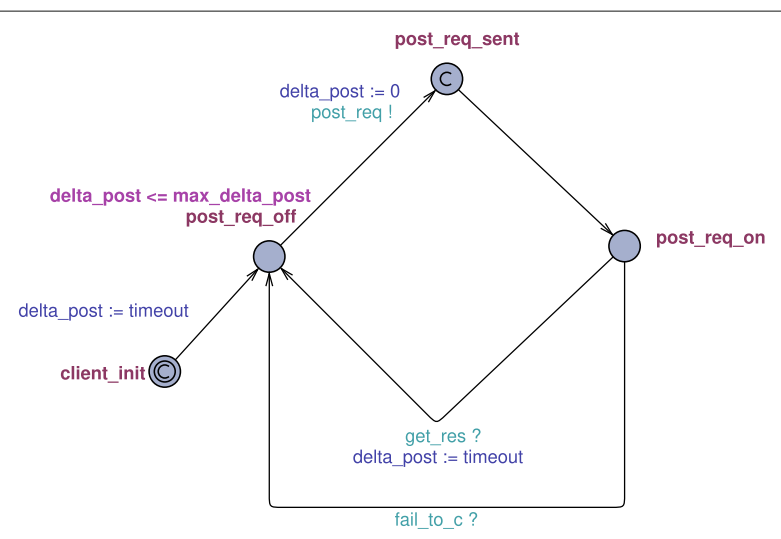

Fig. 10 DeX client automaton

delta_post<=max_delta_post (where max_delta_post is a constant) at the post_req_off location ensures that a new post_req operation will be initiated before the identified boundary.

Based on the above setup, the client sends at most one post_req operation active at a time. This request remains active ( $\delta_{\text {post-req-on }}$ interval) for timeout period (and then it expires) or less than timeout period (in case of successful interaction). In both cases, delta_post equals timeout at the end of the post_req operation. Hence, the immediately following $\delta_{\text {post-off }}$ interval will last a stochastic time uniformly distributed in the interval [ $t$ imeout, max_delta_post]. Such a model is sufficient for verifying the condition (Eq. 3) for successful DeX two-way sync interactions. This condition relates any post_req operation with an overlapping get operation, by taking also into account the deterministic parameter serve_time at the server side.

Figure 11 shows the server behavior. Typically, a server entity repeatedly becomes online (location get_on) to receive requests from the glue. Thus, the server automaton oscillates between the locations get_off and get_on. The get_event committed location is used to detect the online status of the server. It is worth noting that the server entity operates independently from the glue - i.e., it does not notify the glue when changing between the get_on and get_off locations. The automaton stays on the get_on

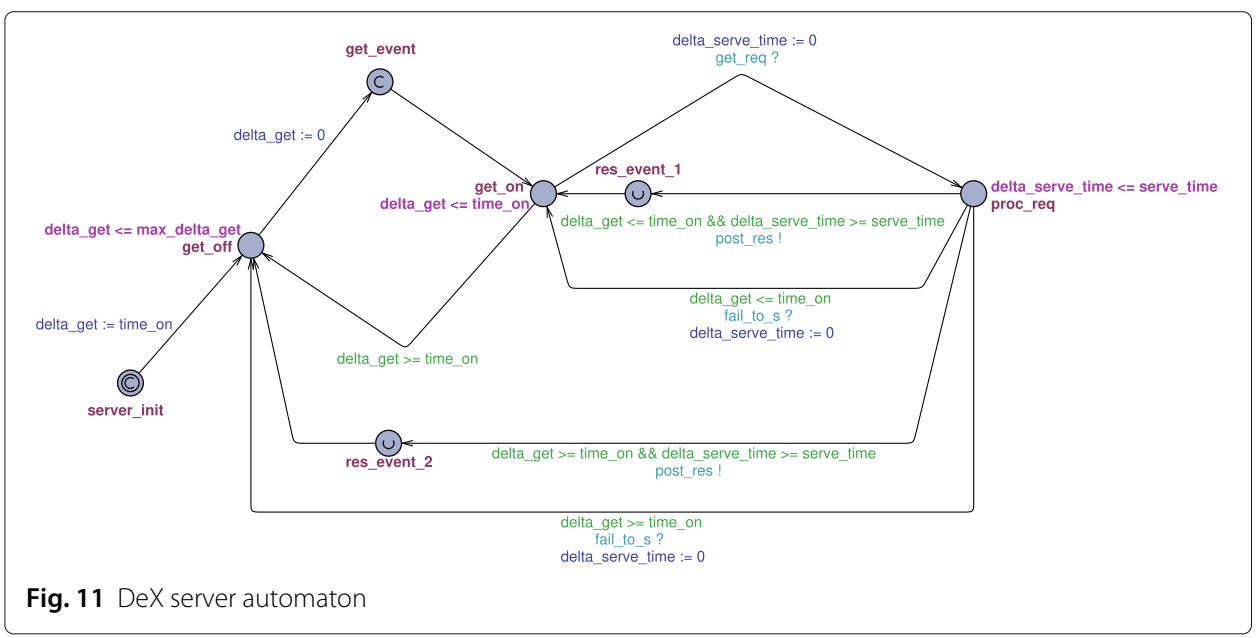


location for a specific interval, which is controlled by the server with a local time_on; upon the time_on, the automaton returns to the get_off location. Similar to the client entity, the delta_get clock is used to measure the time_on interval and switch between the two locations (get_on and get_off). Furthermore, the invariant conditions delta_get<=time_on (at get_on) and delta_get<=max_delta_get (at get_off) guarantee the correct operation of our automaton.

Before reaching the time_on, multiple requests (posted by clients) may be delivered to the server by the glue, each with a get_req? action. Upon a get_req?, the automaton stays in the proc_req location for serve_time interval, which corresponds to the necessary time period for processing a request. We use the urgent res_event_1 and res_event_2 locations to detect successful responses through the post_res! action. Particularly, the res_event_1 location is reached only if the server is still online (delta_get<=time_on). However, while being in location proc_req, the server entity may become offline. For such case, the res_event_2 location is reached after serving the request (because of the invariant delta_serve_time<=serve_time), and then the automaton returns to the get_off location. Finally, the automaton returns to get_on or get_off locations upon a fail_to_s? action received by the glue, which corresponds to the request (post_req) expiration due to the timeout period.

This setup results in a succession of $\delta_{\text {get -req-on }}$ and $\delta_{\text {get-req-off }}$ intervals (see Fig. 6), with the former lasting time_on time and the latter lasting a stochastic time uniformly distributed in the interval [time_on, max_delta_get].

The glue two-way sync automaton is shown in Fig. 12. It determines the synchronization of the incoming (post_req? and post_res?) and outgoing (get_req!) operations. A successful synchronization between such operations leads to a successful interaction, which is represented in the automaton by the trans_succ location. Note that the timing constraints specified in Section 4 regarding the timeout of sent requests have been applied here with the additional clock delta_req_on employed to guard transitions dependent on the timeout period.

The trans_succ location is reached through the following operations: if the post_req? operation occurs from the initial location and the invariant delta_req_on $<=$ timeout is satisfied, a consequent get_req! request is sent to

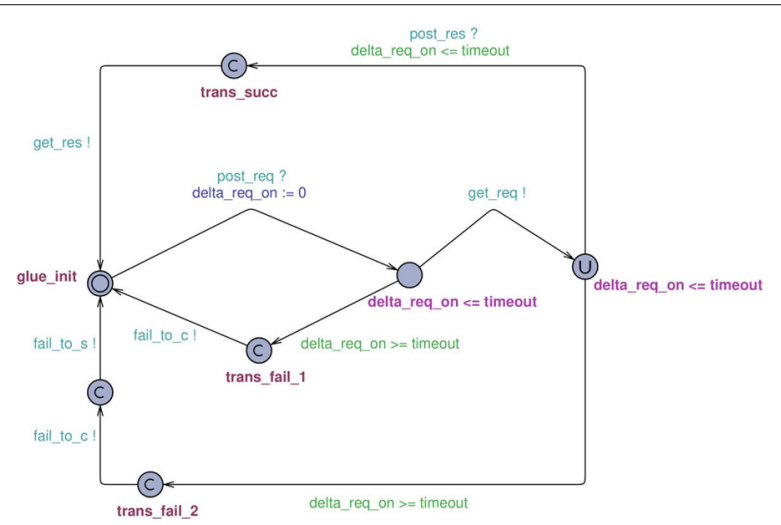

Fig. 12 DeX glue two-way synchronous automaton 
the server (if the server automaton is on the location get_on). While the request is processed on the server side, the glue automaton waits for the reply. After the specified server_time a post_res? operation occurs to the glue and eventually the successful interaction location trans_succ (the Eq. 3 is satisfied). At the same time, the client is notified of the end of the post_req operation with get_res!. Note that we employ the get_req channel as urgent. In this way, upon a post_req? and if the server is online, the get_req! action occurs instantly, without any delay as indicated by the invariant delta_req_on $<=$ timeout.

With regard to the timeout, time_on and serve_time parameters, we identify failed interactions in the glue trough the trans_fail_1 and trans_fail_2. Two ways for reaching the fail locations are considered:

- If the post_req? operation occurs from the initial location and the server automaton is offline (stays on the get_off location) for a time period that leads to the timeout expiration (delta_req_on>=timeout), the trans_fail_1 location is reached. At the same time, the client is notified with fail_to_c! in order to move at the post_req_off location.

- If the post_req? operation occurs from the initial location and the server automaton is online (stays on the get_on location), a consequent get_req! request is sent to the server. While the request is processed for serve_time, the timeout period may expire (delta_req_on $>=$ timeout) and the trans_fail_2 location is reached. At the same time, the client is notified with fail_to_c! to move at the post_req_off location, and the server is notified with fail_to_s! to move either to get_on or to get_off locations, depending of the delta_get clock.

\subsubsection{Verification of properties}

We verify reachability $(\mathrm{E}<>\varphi)$ and safety $(\mathrm{A}[] \varphi)$ properties of the combined automata DeX client, DeX server and DeX two-way sync glue, by using the model checker of UPPAAL. Client Automaton. We verify a set of safety properties that characterize the timings of the client's stochastic behavior.

$$
\begin{aligned}
& \text { A[] client.post_req_sent imply delta_post }==0 \\
& \text { A[] client.post_req_sent imply delta_post }<=\text { timeout } \\
& \text { A[] client.post_req_off imply (delta_post }>=\text { timeout and } \\
& \text { delta_post<=max_delta_post) }
\end{aligned}
$$

Equation 19 states that post_req events occur at time 0 captured by the delta_post clock. Equation 20 states that [0,timeout] is the maximum interval in which a post_req operation is active, nevertheless, the operation can end before timeout is reached. Equation 21 states that [timeout,max_delta_post] is the maximum interval in which there is no active post_req operation. Similar to the $D e X$ sender automaton, we artificially "advance time" to timeout at the end of the post_req operation.

Server Automaton. We verify similar properties that characterize the timings of the server's stochastic behavior. 
A[] server.get_event imply delta_get $==0$

A[] server.get_on imply delta_get<=time_on

A[] server.get_off imply (delta_get>=time_on and

delta_get<=max_delta_get)

Equation 22 states that at the beginning of the server's online period, the automaton passes from the location get_event at time 0 captured by the delta_get clock. Equation 23 states that the server stays online (at the location get_on) at least for time_on interval. Equation 24 states that [time_on,max_delta_get] is the maximum interval in which the server is offline (at the location get_off).

Glue two-way sync Automaton. Finally, we verify conditions for successful interactions using the glue automaton.

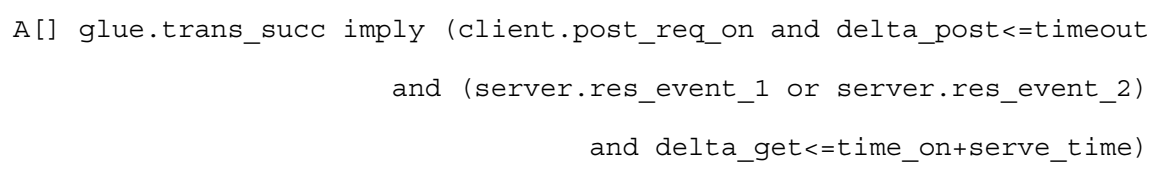

We verify both the reachability property ( $\mathrm{E}<>$ glue.trans_succ) and the safety property in Eq. 25. According to this, a successful interaction event implies that while a post_req operation is active the timeout period is not reached. Additionally on the server side, one of the committed locations res_event_1 or res_event_2 is active and the condition delta_get<=time_on+serve_time holds.

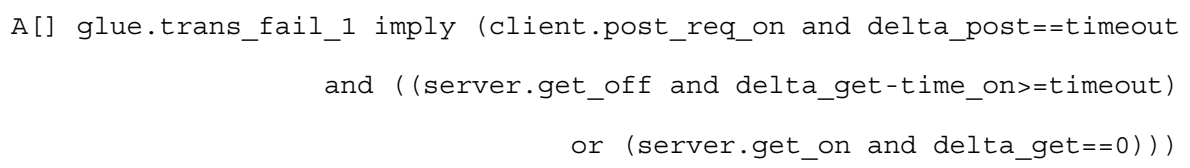

We verify both the reachability property ( $\mathrm{E}<>$ glue.trans_fail_1) and the safety property in Eq. 26. A failed interaction event means that timeout is reached for an active post_req operation. Additionally, the request can not reach the server either because it is offline (get_off location) for time period greater of timeout (delta_get-time_on>=timeout), or due to the fact that the server automaton moved on to location get_on and at the same time the timeout period is reached.

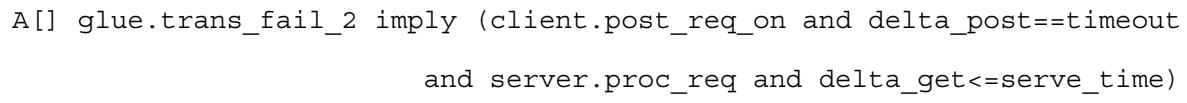

Upon an interaction if the above condition is not verified, it means that the request is processed at the server side. However, an additional failure can occur in location trans_fail_2 while the request is processed. In addition to the reachability property (E<> glue.trans_fail_2), we verify the safety property in Eq. 27. Such a failed interaction event means that timeout is reached for an active post_req operation. Additionally, the request is processed in location proc_req since the condition delta_get $<=$ serve_time is valid. 
Equations 25, 26 and 27 provide us with general formal conditions which can be utilized by system designers to tune timing parameters such as timeout, time_on and serve_time and achieve successful interactions.

\subsection{Analysis of two-Way asynchronous and streaming interactions}

Based on subsections 4.3 and 4.4, the time models for one-way interactions can be leveraged to model the timing behavior of two-way async and streaming interactions. Similarly, the Timed Automata models provided in this section be can be leveraged to derive general formal conditions for successful two-way async and streaming interactions. In particular, we leverage the glue one-way automaton shown in Fig. 9, to derive conditions similar to Eqs. 16, 17, 18 using two-async and streaming DeX operations based on Table 1. For example, in two-way async interactions, we can verify the safety property for successful request transmissions as follows:

A[] glue.trans_succ imply (client.post_req_on and server.get_on and (delta_post_req $==0$ or delta_get $=0$ ) )

According to the above, a successful request transmission implies that while a post_req operation is active a get event occurs at the server side, or while a get operation is active at the server side, a post_req event occurs. Similarly, formal conditions for successful/failed requests, responses, open stream requests and delivery of stream items can be derived. Such conditions can be leveraged by system designers to tune timing parameters.

\subsection{Summary of verification outputs}

In this section, we have provided a detailed view of timed automata modeling, verification and parameter tuning of one-way, two-way and streaming interactions. Having a unified framework for verification allows us to study the effect of timing delays, lifetime parameters and success rates of transmissions. This process does not need to be repeated by users of DeXM within unique deployment scenarios. The verified properties demonstrate that the get and post operations can be managed in a safe way via the timing constraints provided. While we have concentrated on safety and reachability properties composing the ordering of event operations, the models may be reused to verify other associated properties. This formalizes the notion of successful interactions within DeXM.

\section{Simulation-based analysis}

In this section, we provide results of simulations of DeX one-way interactions with varied lifetime and time_on periods. We demonstrate that varying these periods has a significant effect on the rate of successful interactions. Furthermore, the trade-off involved between delivery success rates and response times (depending on lifetime/time_on periods) is evaluated. Finally, we validate our simulation-based analysis by using the DeXM framework which provides implementations of the DeX interactions through real middleware protocols.

Table 3 provides an overview of the experimental settings used in the simulations. These settings are derived from working with real deployments of Middleware such as Java Messaging Service and DPWS. 
Table 3 Experimental settings

\begin{tabular}{|c|c|c|}
\hline Parameter(s) & Definition/Description & Experimental Settings \\
\hline$t_{\text {post }}, t_{\text {get }}$ & $\begin{array}{l}\text { at each timestamp }(t) \text { one post or get } \\
\text { occur. }\end{array}$ & $\begin{array}{l}\text { Poisson arrival rate for } t_{\text {post }} \\
\text { Exponential inter-arrival time for } \\
t_{\text {get. }}\end{array}$ \\
\hline$\delta_{\text {post }}, \delta_{\text {get }}$ & $\begin{array}{l}\text { the time period between two successive } \\
\text { post or get operations. }\end{array}$ & $\begin{array}{l}\text { Exponential distribution for } \delta_{\text {post }} \\
\text { with mean } 10 \mathrm{sec} \text {; } \text { Exponential } \\
\text { distribution for } \delta_{\text {get }} \text { with mean } \\
\text { between } 10 \mathrm{sec} \text {. to } 40 \mathrm{sec} \text {. }\end{array}$ \\
\hline lifetime & message availability/validity in time. & 0 sec. to 40 sec. \\
\hline time_on, time_off & $\begin{array}{l}\text { connected (ON) and disconnected (OFF) } \\
\text { instantaneous periods for receiving } \\
\text { messages. }\end{array}$ & $\begin{array}{l}\text { time_on between } 10 \text { sec. to } 60 \\
\text { sec. }\end{array}$ \\
\hline timeout & $\begin{array}{l}\text { required time period to complete a } \\
\text { request-response synchronous interaction. }\end{array}$ & Derived from the measurements. \\
\hline
\end{tabular}

\subsection{Delivery success rates}

In order to test the effect of varying lifetime and time_on periods on interaction success rates, we perform simulations over the timing analysis one-way model described in Section 4. Poisson arrival rates are assumed for subsequent $t_{\text {post }}$ instances (hence, $\delta_{\text {post }}$ follows the corresponding exponential distribution). Each message is valid for a deterministic lifetime period and then discarded. Similarly, there are exponential intervals between subsequent $t_{\text {get }}$ periods ( $\delta_{\text {get }}$ follows this distribution). The receiver entity is active for a deterministic time_on period and can disconnect for random valued intervals. Applying the one-way timing model in Section 4, the simulation enables concurrent posts with no-queueing. As the arrivals follow a Poisson process, this simulates an $\mathrm{M} / \mathrm{G} / \infty / \infty$ queueing model.

The simulations done in Scilab ${ }^{1}$ analyze the effect of varying li fet $i$ me and time_on periods on DeXM interactions. We set $\delta_{\text {post }}$ between subsequent post messages correspond to post operations presented in 5.1) to have a mean of $10 \mathrm{sec}$. Note that an IoT designer can select different parameters for the subsequent post messages. Nevertheless, in this case the lifetime and time_on periods should carefully be selected in order to highlight their effect on system tuning. The get messages correspond to get operations presented in 5.1) are simulated with varying exponential active periods $\left(\delta_{\text {get }}\right)$. This procedure was run for $10,000 t_{\text {get }}$ periods (enough runs to converge to the average periods selected) to collect interaction statistics with $95 \%$ confidence interval setting; we applied the formal conditions of subsection 5.1 to control the rates of successful interactions. As depicted in Fig. 9, a successful synchronization between post and get operations leads to a successful DeX interaction, which is represented in the automaton by the trans_succ location. The experiments were conducted over a Linux machine with Intel Core i7, 16 Gb RAM.

The rates of successful interactions are shown in Fig. 13 for various values of lifetime, time_on and $\delta_{\text {get }}$ periods. As expected, increasing time_on periods for individual lifetime values improves the success rate. However, notice that the success rate is severely bounded by $l$ ifet $i$ me periods. For instance, when the lifet ime period is very low $(0 \mathrm{sec})$, the success rate, even at higher time_on intervals, remains bound at around $70 \%$ for $\delta_{\text {get }}$ with mean $40 \mathrm{sec}$. Such behavior represents time/space coupled CS interactions, where each message is received immediately by the client (we assume 


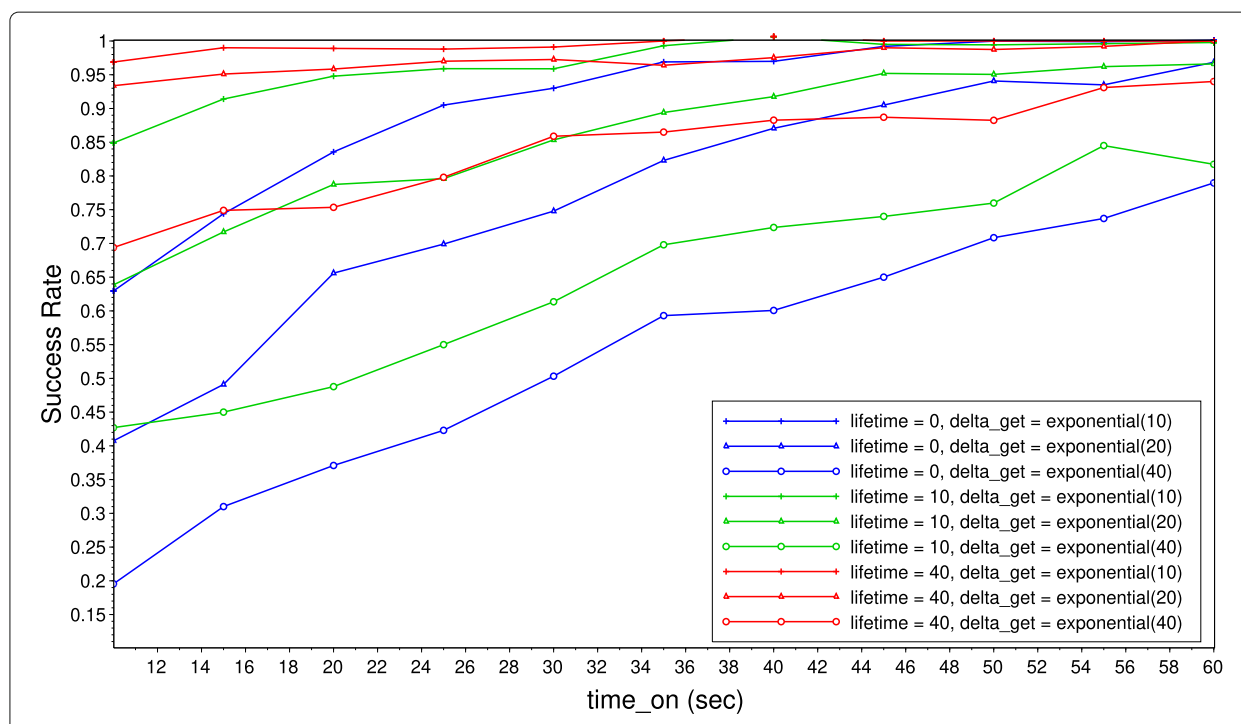

Fig. 13 Delivery success rates with varying time_on and lifetime periods

that the transmission delay of the underlying network delay is negligible). Reducing get disconnection intervals (by properly setting $\delta_{\text {get }}$ and time_on) produces a significant improvement in the success rate, especially for the CS case. For the other interaction paradigms (PS/TS employing an intermediate middleware node), where the lifetime period can be varied: a higher lifetime period combined with higher time_on or lower $\delta_{\text {get }}$ intervals would guarantee better success rates.

\subsection{Response time vs. delivery success rate}

In order to study the trade-off between end-to-end response time and delivery success rate, we present cumulative response time distributions for interactions in Fig. 14. Note that we assume that all posts arriving during an active get period are immediately served;

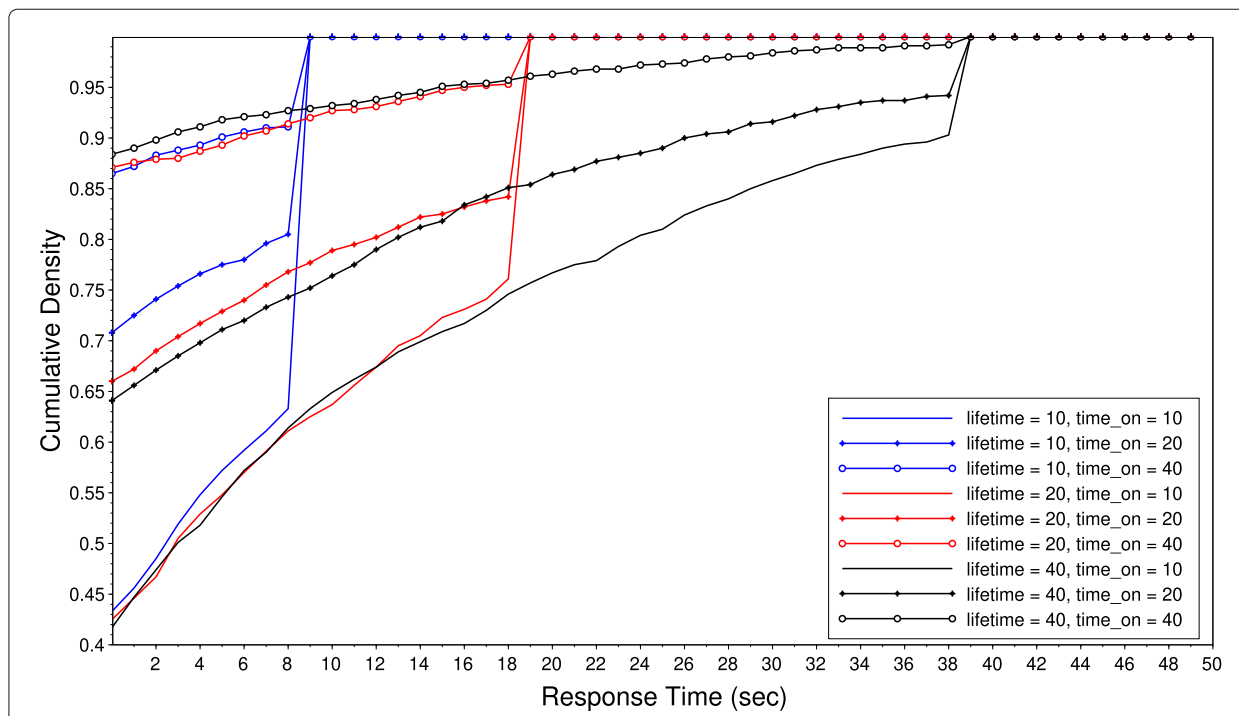

Fig. 14 Response time distributions for interactions with varying time_on and lifet ime periods 
all posts arriving during an inactive get period are immediately served at the next active period, unless they have expired before. All failed transactions are pegged to the value: lifetime.

We set $\delta_{\text {post }}=$ Poisson $(10)$ sec and $\delta_{\text {get }}=$ Exponential(20) sec for all simulated cases. From Fig. 14, lower lifetime periods produce markedly improved response times. For instance, with lifetime $=10 \mathrm{sec}$, time_on $=20 \mathrm{sec}$, all interactions complete within $10 \mathrm{sec}$. Comparing this to Fig. 13, the success rate with these settings is 78\%. Changing to lifetime $=40 \mathrm{sec}$, time_on $=20 \mathrm{sec}$, we get a success rate of $95 \%$, but with increased response time. So, with higher levels of lifetime periods (typically PS/TS), we notice high success rates, but also higher response time. While individual success rates and response time values depend also on the network/middleware efficiency, our analysis provides general guidelines for setting the lifetime and time_on periods to ensure successful interactions.

\subsection{TIM system tuning}

Our fine-grained timing analysis can be employed to properly configure the TIM system. Accordingly, vehicle-devices and fixed-sensors emit posts carrying traffic-related messages with a mean arrival rate of 1 event every 10 min. To guarantee the freshness of provided information, notifications are maintained by the system for a lifetime period of $10 \mathrm{~min}$. We assume that smartphone-users access the system every $20 \mathrm{~min}$ on average to receive up-to-date transport information on their hand-held devices. They stay connected for a time_on period and then disconnect, also for resource saving purposes. Actual connection/disconnection behavior is based on the user's profile. By relying on our statistical analysis, an application designer may configure the time_on period of user access to $10 \mathrm{~min}$. Using scaled values from Figs. 13 and 14 , this guarantees that the user will receive on average $65 \%$ of the posted notifications, within at most $8 \mathrm{~min}$ of response time with a probability of 0.63 . If these values are insufficient and the designer re-configures the time_on to $20 \mathrm{~min}$, this guarantees that now the user will receive on average $80 \%$ of the posted notifications within at most $4 \mathrm{~min}$ of response time with a probability of 0.77 .

\subsection{Comparison with deXM implementation}

In order to validate the simulations performed in Section 6.1, we implement realistic interactions using the DeXM framework. As depicted in Fig. 15, we create two pairs (sender-receiver) of mockup mobile IoT devices using different middleware protocols. In particular, we use two middleware implementations: $(i)$ for lifetime $=0$ transactions, the DPWS ${ }^{2}$ CS middleware provides an API to set a sender and a receiver interacting with each other directly; and (ii) for (lifetime $>0$ ) transactions, the JMS ${ }^{3}$ PS middleware provides an API to set a sender, a receiver, and the intermediate entity through which they interact. Applying the same settings as in Section 6.1, senders and receivers perform operations based on probability distributions (i.e., exponential $\delta_{\text {post }}$ with mean of $10 \mathrm{sec}$ and $\delta_{\text {get }}$ with various mean periods). At the intermediate entity we set various lifetime periods, using the JMS API. All the interactions are performed using an Intel Xeon W3550e $3.08 \mathrm{GHz} \times 4$ (7.8GB RAM) under a Linux Mint OS. Note that 
(Inte|Xeon W3550e 3.08 GHz×4
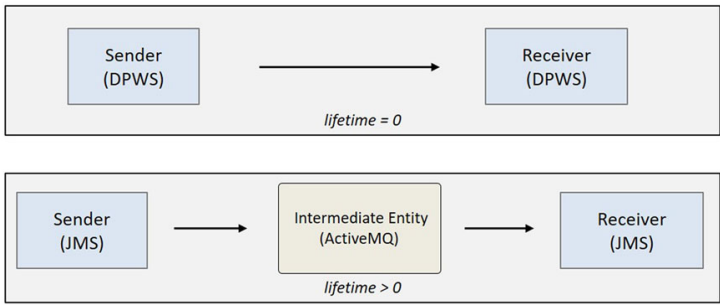

Fig. 15 DeXM experimental setup

in these DeXM implementation settings, we have concurrent posts and queueing. This corresponds to an $\mathrm{M} / \mathrm{G} / 1 / \infty$ queueing model; however, the queueing time of data due to processing of preceding data is negligible in our specific settings.

For getting reliable results, the mean values of $\delta_{\text {post }}$ and $\delta_{\text {get }}$ intervals are expected to be close to the expected mean values. To do so, we create sufficient number of post operations and get connections/disconnections by running each experiment for at least 2 hours. In Table 4, we compare the results of simulated and measured success rates for time_on $=20 \mathrm{sec}, \delta_{\text {post }}=$ Poisson(10) sec, lifetime $=0,10,40 \mathrm{sec}$ and various distributions for $\delta_{\text {get }}$. The absolute deviation between the two is no more than $10 \%$. This deviation may be attributed to implementation factors such as buffering at each entity (sender, receiver, intermediate entity) which may affect the success rates. As this deviation is not too high, it allows developers to rely on our simulation model to tune the system.

\section{Related work}

IoT devices exchange data determined by functional semantics such as space, time and synchronization coupling. In the mobile IoT, the data exchange is highly dependent on time semantics such as data availability/validity, intermittent connectivity and applayer processing time. Consequently, investigating generic evaluation techniques of such system semantics is crucial.

In [19], the authors express the intermittent WiFi availability for a mobile user using 2-D Markov chains. This is a complex and tedious procedure. Extending this approach to mobile peers of middleware systems (e.g., publish/subscribe) can be even more complicated, due to the combined modeling of network-level and application-level time semantics. Alternatively, Queueing Network (QNs) and Performance Petri Nets (PPNs) are both "high level" flexible techniques for describing (primarily) Markov models which can be used for constructing performance metrics about computer systems and, subsequently, middleware systems [20]. The notation used to describe the models enables the user

Table 4 Simulated vs. measured delivery success rates

\begin{tabular}{llll}
\hline lifetime $(\mathbf{s})$ & $\boldsymbol{\delta}_{\text {get }}(\mathbf{s})$ & Simulation & Measurement \\
\hline 0 & exponential (20) & 0.65 & 0.717 \\
0 & exponential (40) & 0.35 & 0.42 \\
10 & exponential (20) & 0.75 & 0.778 \\
10 & exponential (40) & 0.48 & 0.554 \\
40 & exponential (20) & 0.93 & 0.91 \\
40 & exponential (40) & 0.75 & 0.81 \\
\hline
\end{tabular}


to develop and explore a large design space rapidly. Along the dimensions of expressive power and solution efficiency, PPNs enjoy an advantage over QNs in representing synchronization (parallel systems) and are probably best suited for design purposes. A closely related work is [13], where formal analysis (using colored Petri-Nets) of various types of time synchronization in distributed middleware architectures has been performed. On the other hand QNs provide convenient primitives for constructing models, guarantee that are well-formed (i.e., stable, deadlock-free, etc), and can be solved efficiently. Work done by Kattepur and Nambiar [21] makes use of QNs to estimate performance of Web applications using algorithms such as Mean Value Analysis (MVA).

In comparison to the above approaches that are used to model and analyze queueing and synchronization phenomena of systems, we model DeX interactions (which unify heterogeneous underlying mobile IoT interactions) by focusing on the high-level timing semantics of mobile peers. This combines network-level and application-level semantics, while assuming that the message processing, transmission and queueing times inside an interaction are negligible compared to the timing behaviors of mobile peers. Hence, we opt for employing statistical modeling, simulation and subsequent analysis for deriving general performance metrics for heterogeneous mobile IoT systems.

On the other hand, timed automata [5] can be used to model and analyze the formal timing behavior of computer systems, e.g., real-time systems or protocols. They have been applied to a variety of real time system models to ensure accurate behavior under timed guards. Such models enable checking both safety and liveness properties and they have been particularly developed and studied over the last years. Model checkers such as UPPAAL [6], PRISM [22] and SBIP [23] have been proposed for analyzing timed and probabilistic properties of systems. Timed automata are used in [24] for studying fault tolerant behavior (safety, bounded liveness) in distributed asynchronous real time systems. Furthermore, in [25], a hierarchical timed automata based approach is proposed to model and analyze the dynamic software evolution of service oriented systems. Both functional evolution (with structural changes of the software architecture) and non-functional evolution (with parameter changes) are considered. Hierarchical timed automaton (HTA) introduces a refinement function to map a composite location to an underlying set of automata, and hence can describe the hierarchical structure of service composition.

In [26], the transmission channels of publish/subscribe middleware as well as overlying application components are modeled using probabilistic timed automata for verifying properties of the supported interactions with the PRISM probabilistic model checker. The same authors do model-checking of the timed behavior of publish/subscribe applications using the Bogor model checker, which can be customized to different application domains [27]. Finally, in [28], the authors demonstrate the necessity of applying formal models to IoT protocols. In particular, they model the MQTT publish/subscribe protocol based on a timed message-passing process algebra. The analysis reveals that the protocol behaves correctly regarding the semantics of the QoS modes 1 and 2. However, the 3rd QoS mode is prone to error and at best ambiguous in certain aspects of its specification.

Our work relies on some of the formal modeling and analysis techniques of the above approaches. We further introduce a new timed model that abstracts the timing behavior of mobile IoT interactions across the existing heterogeneous IoT protocols. Our formal analysis based on timed automata and UPPAAL provides general conditions for successful interactions independently of the diverse underlying IoT protocols. 
Alternatively or in addition to simulation based approaches, statistical model checking [29] may be applied in order to verify, for instance, probabilistic reachability properties. Nevertheless, simulation techniques are necessary as a starting point in order to elicit distributions needed as inputs to statistical model checkers. This is the case in [29], where authors perform simulations of a system in order to learn the application context. This creates a stochastic abstraction for the application, which is verified using statistical model checking. In the work done by Kim et al. [30], a formal specification is developed for each layer of a distributed system. To achieve the desired end-to-end timing/QoS properties, the formal specification is analyzed using statistical model checking and statistical quantitative analysis under various resource management policies. In our approach, we employ statistical quantitative analysis for evaluating performance properties of mobile IoT interactions by calculating, e.g., their mean values or probability distributions. Statistical model checking can be our next step for verifying or estimating the probabilities of such properties.

Overall in this paper, we unify the verification of the timing behaviors of DeX one-way, synchronous, asynchronous and streaming interactions, as well as the statistical performance analysis of such interactions. While our prior work focused on the timing and QoS analysis of heterogeneous service choreographies [4,31], we model here the finegrained effect of timing parameters on both coupled and decoupled mobile IoT systems. By leveraging our analysis of timing parameters, designers of IoT systems can accurately set constraints to ensure high success rates for interactions.

\section{Conclusion and future work}

Timing constraints have typically been used for time-sensitive systems to ensure properties such as deadlock freeness and time-bounded liveness. In this paper, we study IoT interactions using the DeX connector model which is enhanced to accurately model timing behavior through timed automata. Verification of conditions for successful DeX interactions is done in UPPAAL in conjunction with the timing guards specified. We demonstrate that accurate setting of lifetime, timeout, time_on and serve_time periods significantly affects the DeX interactions success rate. By providing a fine-grained analysis of the related timing parameters for designers of IoT applications, increased probability of successful interactions can be ensured. This is crucial for accurate runtime behavior, especially in the case of heterogeneous space-time coupled/decoupled DeX interactions with variable connectivity of IoT devices. Furthermore, we demonstrate that the response time vs. success rate trade off can be suitably configured. Finally, we confirm the sufficient accuracy of our results by comparing with experimental outcomes from the DeXM implementation framework.

The timed modeling and analysis of IoT interactions presented in this paper can open up directions for the design of robust and efficient IoT systems. In our future work, we intend to model the performance of realistic DeX interactions by considering processing and transmission delays, as well as the effect of DeXM mediators. We will leverage our experience in the modeling of publish/subsbribe protocols using queueing network models. Such models can provide us with both simulation and analytical models for estimating performance metrics such as response times and delivery success rates. Finally, these performance metrics can be leveraged for runtime system tuning and reconfiguration using heuristic and optimization techniques. 


\begin{abstract}
Abbreviations
IOT: Internet of things; APIs: Application programming interfaces; DeX: Data eXchange; DeXM: Data eXchange mediators; SOA: Service-oriented architecture; CS: Client/server; PS: Publish/subscribe; DS: Data streaming; TS: Tuple space; TIM: Transport information management; QoS: Quality of service; RPC: Remote procedure call; CTL: Computation tree logic; DPWS: Devices profile for web services; JMS: Java message service; QNs: Queueing networks; PPNs: Performance petri nets; MVA: Mean value analysis; HTA: Hierarchical timed automaton
\end{abstract}

\title{
Acknowledgements
}

We acknowledge colleagues of the INRIA MINES team for their suggestions.

\section{Funding}

This work has been partially supported by the European Union's Horizon 2020 project CHOReVOLUTION under grant agreement No. 644178.

\section{Availability of data and materials}

Our prototype and its modules are publicly available at: https://gitlab.inria.fr/dexms

\section{Authors' contributions}

All authors read and approved the final manuscript.

\section{Declarations}

\section{Competing interests}

The authors declare that they have no competing interests.

\section{Author details}

${ }^{1}$ Télécom SudParis, Institut Polytechnique de Paris, France. ${ }^{2}$ INRIA, Paris, France. ${ }^{3}$ Ericsson Research, Bangalore, India.

\section{Received: 19 April 2020 Accepted: 20 October 2021}

Published online: 01 December 2021

\section{References}

1. Guinard D, Trifa V, Karnouskos S, Spiess P, Savio D. Interacting with the soa-based internet of things: Discovery, query, selection, and on-demand provisioning of web services. IEEE Trans Serv Comput. 2010;3(3):223-35.

2. Fysarakis K, Askoxylakis I, Soultatos O, Papaefstathiou I, Manifavas C, Katos V. Which iot protocol? comparing standardized approaches over a common m2m application. In: IEEE Global Communications Conference (GLOBECOM). Washington: IEEE; 2016.

3. Bouloukakis G, Georgantas N, Ntumba P, Issarny V. Automated synthesis of mediators for middleware-layer protocol interoperability in the iot. Futur Gener Comput Syst. 2019;101:1271-94. https://doi.org/10.1016/j.future.2019.05.064.

4. Kattepur A, Georgantas N, Bouloukakis G, Issarny V. Analysis of timing constraints in heterogeneous middleware interactions. In: ICSOC. Goa: Springer; 2015.

5. Alur R, Dill DL. A theory of timed automata. Theor Comput Sci. 1994;126(2):183-235.

6. Behrmann G, David A, Larsen KG. A tutorial on Uppaal 4.0: Department of computer science, Aalborg university; 2006.

7. Schrank D, Eisele B, Lomax T. Tti's 2012 urban mobility report. 2012.

8. ITS. Intelligent Transportation Systems. http://www.flir.co.uk/traffic/content/?id=66601.

9. XD. Traffic. http://inrix.com/xd-traffic.

10. Yoon J, Noble B, Liu M. Surface street traffic estimation. In: ACM Mobisys. PR: ACM; 2007.

11. Mohan P, Padmanabhan VN, Ramjee R. Nericell: rich monitoring of road and traffic conditions using mobile smartphones. In: ACM SenSys. Raleigh: ACM; 2008.

12. Eugster P, Felber P, Guerraoui R, Kermarrec A. The many faces of publish/subscribe. ACM Comput Surv (CSUR). 2003;114-31.

13. Aldred $L$, van der Aalst W, Dumas $M$, ter Hofstede A. On the notion of coupling in communication middleware. In: OTM Confederated International Conferences" On the Move to Meaningful Internet Systems". Agia Napa: Springer; 2005.

14. Georgantas N, Bouloukakis G, Beauche S, Issarny V. Service-oriented Distributed Applications in the Future Internet: The Case for Interaction Paradigm Interoperability. In: ESOCC. Managa: Springer; 2013.

15. Bouloukakis G, Moscholios I, Georgantas N, Issarny V. Performance modeling of the middleware overlay infrastructure of mobile things. In: IEEE ICC. Paris: IEEE; 2017.

16. Bouloukakis G, Georgantas N, Kattepur A, Issarny V. Timeliness Evaluation of Intermittent Mobile Connectivity over Pub/Sub Systems. In: ACM/SPEC ICPE. L Aquila: ACM; 2017.

17. Bouloukakis G, Kattepur A, Georgantas N, Issarny V. Queueing network modeling patterns for reliable and unreliable publish/subscribe protocols. In: 15th EAl International Conference on Mobile and Ubiquitous Systems: Computing, Networking and Services (MobiQuitous). New York: ACM; 2018.

18. Clarke E, Emerson A, Sistla P. Automatic verification of finite-state concurrent systems using temporal logic specifications. ACM Trans Program Lang Syst (TOPLAS). 1986;8(2):244-63.

19. Lee K, Lee J, Yi Y, Rhee I, Chong S. Mobile data offloading: how much can wifi deliver?. In: Proceedings of the 6th International COnference. ACM; 2010.

20. Vernon M, Zahorjan J, Lazowska ED. A Comparison of Performance Petri Nets and Queueing Network Models: University of Wisconsin-Madison, Computer Sciences Department; 1986. 
21. Kattepur A, Nambiar M. Performance modeling of multi-tiered web applications with varying service demands. In: IPDPS Workshops. Hyderabad: IEEE; 2015.

22. Kwiatkowska M, Norman G, Parker D. PRISM: Probabilistic symbolic model checker. In: MMMECCS. Aachen: Springer; 2001.

23. Nouri A, Bozga M, Legay A, Bensalem S. Performance evaluation of complex systems using the sbip framework. In: VECOS. Montreal: Springer; 2016

24. Waszniowski L, Krakora J, Hanzalek Z. Case study on distributed and fault tolerant system modeling based on timed automata. J Syst Softw. 2009.

25. Zhou Y, Ge J, Zhang P, Wu W. Model based verification of dynamically evolvable service oriented systems. Sci China Inf Sci. 2016.

26. He F, Baresi L, Ghezzi C, Spoletini P. Formal analysis of publish-subscribe systems by probabilistic timed automata. In: FORTE. Tallinn: Springer; 2007.

27. Baresi L, Ghezzi C, Mottola L. On accurate automatic verification of publish-subscribe architectures. In: ICSE. Minneapolis: IEEE Computer Society; 2007.

28. Aziz B. A formal model and analysis of an iot protocol. Ad Hoc Netw. 2016;36:49-57.

29. Basu A, Bensalem S, Bozga M, Caillaud B, Delahaye B, Legay A. Statistical abstraction and model-checking of large heterogeneous systems. 2010.

30. Kim M, Stehr MO, Talcott C, Dutt N, Venkatasubramanian N. Combining formal verification with observed system execution behavior to tune system parameters. Quebec: Springer; 2007.

31. Kattepur A, Georgantas N, Issarny V. Qos analysis in heterogeneous choreography interactions. In: International Conference on Service-Oriented Computing. Springer; 2013.

\section{Publisher's Note}

Springer Nature remains neutral with regard to jurisdictional claims in published maps and institutional affiliations.

\section{Submit your manuscript to a SpringerOpen ${ }^{\circ}$ journal and benefit from:}

- Convenient online submission

Rigorous peer review

- Open access: articles freely available online

- High visibility within the field

- Retaining the copyright to your article

Submit your next manuscript at $\boldsymbol{\triangleright}$ springeropen.com 